\title{
Intelligent Reflecting Surface Empowered Wireless Powered Caching Networks
}

\author{
Zheng Chu, Member, IEEE, Pei Xiao, Senior Member, IEEE, Mohammad Shojafar, Senior Member, IEEE \\ De Mi, Member, IEEE, Wanming Hao, Member, IEEE, Jia Shi, Member, IEEE, and Jie Zhong, Member, IEEE
}

\begin{abstract}
In this paper, we propose an intelligent reflecting surface (IRS) enabled wireless powered caching system. In the proposed IRS model, a power station (PS) provides wireless energy to multiple Internet of Things (IoT) devices, delivering their information to an access point (AP) by utilizing the harvested power. The AP, equipped with a local cache, stores the IoT data to avoid waking up the IoT devices frequently. Meanwhile, we deploy the IRS involving in the wireless energy and information transfer process for performance enhancements. In this practical system, the PS and the AP could belong to different service providers. Also, the AP requires to incentivize the PS to offer a provisional energy service. We model the interaction between the PS and the AP as a Stackelberg game that jointly optimizes the transmit power of the PS, the energy price, the phase shifts of the wireless energy transfer (WET) and wireless information transfer (WIT) phases, as well as wireless caching strategies of the AP. In this way, we first derive the optimal solutions of the phase shifts and the transmit power of the PS in closed-form. We propose an alternating optimization (AO) algorithm to optimize the wireless caching strategies and the energy price iteratively. Finally, we present various numerical evaluations to validate the beneficial role of the IRS and the wireless caching strategies and the performance of the proposed scheme compared with the existing benchmark schemes.
\end{abstract}

Index Terms-Intelligent reflecting surface (IRS), wireless caching, wireless powered communication networks (WPCN), Internet of Things (IoT), phase shifts, Stackelberg game.

\section{INTRODUCTION}

Wireless sensing and massive connectivity have been regarded as the most significant components in Internet of Things (IoT) networks, which has attracted an increasing attention in academia and industry. In an IoT network, the wireless devices (WDs) deliver their own sensed data to the access points (APs) for various practical applications, e.g., environment monitoring, wireless surveillance, etc. [1]. Typically, the APs collect these sensed data and then transmit them to users via backhaul links upon their own requests [2]. An explosive growth of the IoT service demand has

This work was supported in part by the Engineering and Physical Sciences Research Council (EPSRC) Project under Grant EP/P03456X/1.

Z. Chu, P. Xiao, M. Shojafar, and D. Mi are with 5GIC \& 6GIC, Institute for Communication Systems (ICS), University of Surrey, Guildford GU2 7XH, UK. (Email: andrew.chuzheng7@gmail.com, p.xiao@surrey.ac.uk, m.shojafar@surrey.ac.uk,d.mi@surrey.ac.uk)

W. Hao is with the School of Information Engineering, Zhengzhou University, Zhengzhou 450001, China. (Email: iewmhao@zzu.edu.cn)

J. Shi is with The State Key Laboratory of Integrated Service Net- works, Xidian University, Xian 710071, China. (Email: jiashi@xidian.edu.cn)

J. Zhong is with the College of Information Science and Electronic Engineering, Zhejiang University, Hangzhou 310027, China. (Email: zhongjie@zju.edu.cn) caused a dramatic traffic increase in the IoT network, which degrades the WDs' quality of service (QoS). In addition, energy constraint is a major issue for those WDs, limiting their capabilities. Although various research contributions have been made to the energy efficient management policies, the lifetime of the WDs still remains an unconquered challenge [3].

Recently, wireless powered communication networks (WPCNs) have emerged as a promising solution to address the energy constraint issue by utilizing electromagnetic transmission. WPCNs are originated from the radio frequency (RF) wireless energy transfer (WET) to ensure a stable energy supply [4]-[6]. In a generic WPCN, a classic transmission policy, "harvest-then-transmit", was adopted. The WDs first collect energy from a hybrid AP and then utilize harvested power for data transmission [7]. The main advantages of WPCNs include mitigating the circuit operational cost and enhancing the sustainability of the WDs, which is a viable solution for the low-energy consumption sensors.

Although the existing advanced techniques have been considered to enhance the capacity of wireless networks [8], [9], these techniques require a large amount of RF chains for data transmission over a high frequency band which consumes more energy and increases hardware costs. This imposes a new challenge since more emission of radio waves is needed to transmit more data [10]-[12]. To deal with this challenge, a novel and promising paradigm i.e., intelligent reflecting surfaces (IRS), has been considered as one of the most revolutionized techniques to effectively improve the spectral/energy efficiency of wireless networks [13] The IRS is made up of a large number of programmable reflecting elements, managed by a smart controller. Each element features small-size, lowcost, and low-power consumption, and induces an adjustable amplitude or phase which can be intelligently tuned to coordinate the incident signal [14].

On the other hand, an increasing amount of IoT sensed data are transmitted to the mobile users inducing explosive growth in IoT traffic and causing WDs to consume more and more energy since they are expected to deliver IoT data to the mobile users frequently [2]. To cope with this issue, wireless caching has been developed to reduce the data traffic as well as energy consumption. Local cache is attached in the AP, which pre-fetches the most popular data from the WDs and store them in its local cache [15]. If the data requested by the users are locally cached in the AP, then they can be directly delivered from the AP to the users via the backhaul link rather than frequently keeping these WDs active [16]. Thus, 
the optimal caching strategies should be designed which aims to determine which data are appropriate for caching to take full advantage of the finite cache capacity to enhance wireless network performance.

\section{A. State-of-the-art}

1) IRS Assisted Wireless Networks: Recently, various research contributions have been made to apply IRS to different wireless networks [13], [17]-[25]. In [13], [17], the active transmit beamforming and passive reflecting beamforming were characterized in an IRS enabled multiple-input singleoutput (MISO) downlink system. Also, the IRS has been investigated to improve wireless security performance in [18]-[20]. Power efficiency and achievable secrecy rate were optimized via designing the active secure transmit and passive reflecting beamformers, alternatively [18], [19]. In [20], artificial noise (AN) was proved to be beneficial for the secrecy rate of the IRS assisted security system compared with the counterparts without IRS or AN. The IRS assisted multi-cell multipleinput multiple-output (MIMO) system was considered in [21], where the edge users take full advantage of such a system deployment to suppress the inter-cell interference. The IRS has been recently considered in simultaneous wireless information and power transfer (SWIPT) to improve the energy harvesting and data communication capabilities [22]-[24]. Specifically, In [22], the WET efficiency was enhanced and the rateenergy trade-off is characterized to maximize the weighted harvested power. Also, the minimization problem of the total transmit power is iteratively solved by an efficient penaltybased method, which demonstrates the effect of IRSs on the energy efficiency enhancement [23]. In [24], an IRS assisted MIMO SWIPT system was exploited to maximize the WSR to guarantee the energy harvesting requirement. Very recently, the IRS have been applied to the mmWave communications over orthogonal frequency division multiple access (OFDMA) in [25]. On the other hand, the IRS has been considered to benefit the uplink transmission, i,e., the IRS assisted multiple access channel (MAC) [26], and the IRS assisted WPCN with user cooperation (UC) [27]. The centralized and distributed IRSs were considered to exploit capacity region of the uplink MAC and analyze deployment mechanism of the IRS [26]. The authors in [27] investigated a two-user UC assisted WPCN to exploit the beneficial role of the IRS in improving energy harvesting and data transmission capabilities. Very recently, the IRS assisted mobile edge computing (MEC) were developed, which aims to maximize sum computational bits to exploit the beneficial role of the IRS on the overall computing performance of the MEC system [28].

2) Caching Assisted Wireless Networks: In recent years, wireless caching has been considered as one of most important techniques to improve QoS of users as well as reduce the costs of wireless network operations, which has attracted more and more research attentions [29]. An increasing number of research contributions have been made in existing works that mainly focus on wireless caching strategies [30]-[35]. In [30], a content-centric transmission mechanism was proposed in a cloud radio access network (C-RAN). Specifically, the same content is requested by multiple users which are cooperatively served by a group of BSs. Local cache is attached in each BS which requests the contents either from its local cache or from the central processor via backhaul connections. The power control scheme was proposed in the NOMA assisted wireless caching networks [31], where an iterative algorithm was proposed to design the optimal power allocation to minimize the transmission delay via a deep neural network (DNN). Moreover, the cache-enabled network has been considered to reduce the delivery latency [32], where the efficient algorithms were designed for the low-latency cloud-edge coordinated transmission strategies. In [33], the performance gain in energy efficiency has been confirmed in the cache-enabled networks. Furthermore, RF WET has been integrated with fog radio access network (F-RAN) to guarantee the downloading requirement of the content users and the wireless charging requirement of the energy users simultaneously [34]. In [35], multiple device-to-device (D2D) pairs provide the contents with caching capacities, where the mobile users access the optimal spectrum to download the contents, and a Q-learning spectrum access scheme is proposed to maximize the transmission rate.

Despite the existing works (including the ones mentioned above) in the downlink/uplink IRS or caching assisted wireless networks, there still exists a significant research gap on an investigation of the IRS assisted wireless powered caching strategies in the case where the IRS assisted WPCN can perform energy harvesting and data transmission enhancements. The IRS can adequately coordinate the energy/information RF signals to offer coverage extension for downlink wireless charging and uplink throughput enhancements for the WPCN. On the other hand, wireless transmission links are sometimes not perfect due to the shadowing or long distance. Also, wireless cache delivery may suffer from performance uncertainties during wireless transmissions, which is imperative to integrate with IRS and WPCN to enhance the energy harvesting, information transmission as well as caching capabilities. To the best of the authors knowledge, there is a lack of work that exploited the IRS assisted wireless powered caching strategies, which motivates this paper.

Motivated by the earlier related works' considerations, this paper exploits the IRS in a wireless powered caching system. For the system under study, we summarize the main contributions as follows:

1) First, we present an IRS assisted wireless powered caching network, where the IRS is deployed to enhance energy harvesting. In such a network, data transmission capabilities and a local cache are attached in the AP to prefetch the popular data sent from the IoT devices.

2) We evaluate the overall performance of the proposed system in a practical and challenging scenario where the WET and WIT networks may belong to different service providers. In this case, an energy incentive mechanism is adopted to stimulate the PS to provide wireless charging, which is modelled as a Stackelberg game between the WET and WIT networks. Specifically, the AP purchases incentives to the PS for wireless charging to the IoT devices, which optimally design the wireless caching 
strategies, energy pricing, and the phase shifts of the WIT phase. We aim to maximize the utility function that is defined as the difference between the benefits obtained from the sum throughput of the data transmission plus the backhaul data rate and the payment for the energy service. The PS determines the optimal solutions of the transmit power of the PS and the phase shifts of the WET phase to maximize its utility function defined as the difference between the energy payment and the energy operation cost, which is typically modelled as a quadratic function.

3) We take into consideration the Stackelberg equilibrium to solve the formulated Stackelberg game. Specifically, we first propose the Majorization-Minimization (MM) and complex circle manifold (CCM) algorithms to optimally derive the closed-form phase shifts of the WET phase. Next, the closed-form transmit power of the PS is derived. Then, we consider an AO algorithm to iteratively design the wireless caching strategies and the energy pricing. Particularly, we first derive the optimal phase shifts of the WIT phase by triangle inequality, and analyze the performance gain brought from the IRS. Then, we optimize caching strategies for given energy pricing, equivalent to solving the $0-1$ knapsack problem. The optimal energy pricing is then designed for given caching strategies.

The rest of this paper is organized as follows. The system model are described in Section III In Section III the Stackelberg game is formulated. Section IV investigates the Stackelberg equilibrium of the formulated game. Section $\nabla$ provides numerical results to evaluate the proposed algorithm. Finally, we conclude this paper in Section VI

\section{System Model}

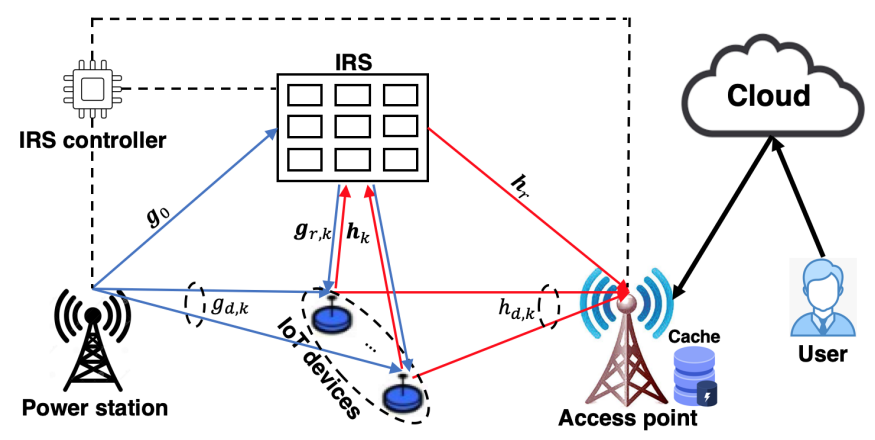

Fig. 1: An IRS assisted wireless powered cache network.

An IRS enabled wireless powered caching network under study is depicted in Fig. 1] where a PS provides wireless energy to $K$ IoT devices which then delivery their own messages to an AP by utilizing harvested power. Meanwhile, an IRS, consisting of $N$ reflecting elements, is deployed to improve energy harvesting and data transmission capabilities via generating passive energy and information passive beamformers. The IRS controller is typically attached to manage its reflection mode via intelligently tuning the phase shift of each reflecting element. The two operation modes of IRS include channel estimation energy/data reflection, and a real-time CSI feedback [13]. It is also assumed that all CSIs follow the quasistatic flat-fading model. In addition, the AP consists of a local cache equipped with a limited storage capacity, which connects to a cloud through a wired backhaul link and transmits the received data from the IoT devices to the users. In this paper, we assume that the IoT data is not always time-sensitive, e.g., free AR/VR content subscriptions of wearable devices and software/firmware update requests of low-cost devices. These IoT data transmitted by several IoT devices can be pre-fetched at the local cache of the AP in the previous time frame according to their own popularity for the users. This can effectively avoid waking up the IoT devices too frequently so as to reduce the latency and energy consumption of these IoT devices. In addition, the time-sensitive requirement could be considered as one of significant metrics in the IRS wireless powered caching network, which is to minimize the latency of information transmission and local wireless caching by jointly designing the IRS phase shifts, time scheduling, and caching strategy. This design would deserve another investigation, which is left for our future work. When the user requests certain IoT data, they are retrieved from the AP's local cache, or the corresponding IoT device directly delivers the data to the user via the AP.

\section{A. Wireless Powered Communication Network}

For the system under investigation, we adopt a generic harvest-then-transmit protocol. The PS first provides WET service to the IoT devices during the downlink energy transmission/reflection duration of $\tau_{0}$. At the same time, the IRS passively reflects the energy signal via its planar arrays to improve energy harvesting capability at the IoT devices. Then, these IoT devices utilize the harvested power for WIT with the help of the IRS. The time division multiple access (TDMA) scheme is adopted during this uplink information transfer/reflection duration, each of which is allocated at $\tau_{k}, \quad k \in[1, K]$. Note that our work mainly focuses on the optimal design of the wireless caching strategies at the AP and the IRS phase shifts. Thus, we consider the equal time scheduling, i.e., $\tau_{0}=\tau_{1}=, \ldots=\tau_{K}$, for convenience and without loss of generality 1 We denote $\boldsymbol{\Theta}_{k}=$ $\operatorname{diag}\left[\beta_{k, 1} \exp \left(j \alpha_{k, 1}\right), \ldots, \beta_{k, N} \exp \left(j \alpha_{k, N}\right)\right], \quad\left|\exp \left(j \alpha_{k, n}\right)\right|=$ $1, \forall k \in[0, K], n \in[1, N]$ as the diagonal phase shift matrices of the IRS for energy/information reflection, where $\alpha_{k, n} \in[0,2 \pi)$ and $\beta_{k, n} \in[0,1]$ denotes the associated phase shift and amplitude 2 When $k=0, \boldsymbol{\Theta}_{0}$ is also known as the energy reflection phase shift matrix, whereas $\Theta_{k}$ is called the information reflection phase matrix when $k \in[1, K]$. In order to maximize the energy and information reflections, the reflecting amplitude of each element on the IRS should be set

\footnotetext{
${ }^{1}$ Joint optimization of the transmission time scheduling, the wireless caching, as well as the IRS phase shifts leads to a more complicated problem formulation and algorithm design, which deserves a separate investigation in our future work.

${ }^{2}$ Practically, the phase shifts are typically selected with a series of finite values from 0 to $2 \pi$ to reduce the complexity of circuit implementation, however, this would lead to a performance degradation [13], [36].
} 
to be one, i.e., $\beta_{k, n}=1, k \in[0, K], n \in[1, N]$ to achieve the maximum reflection gain. We denote the channel coefficients from the PS to the $k$-th IoT device, from the PS to the IRS, from the IRS to the $k$-th IoT device, from the $k$-th IoT device to the AP, from the $k$-th IoT device to the IRS, and from the IRS to the AP as $g_{d, k} \in \mathbb{C}^{1 \times 1}, \mathbf{g}_{0} \in \mathbb{C}^{1 \times N}, \mathbf{g}_{r, k} \mathbb{C}^{N \times 1}$, $h_{d, k} \in \mathbb{C}^{1 \times 1}, \mathbf{h}_{k} \in \mathbb{C}^{1 \times N}$, and $\mathbf{h}_{r} \in \mathbb{C}^{N \times 1}$, respectively. Thus, the RF harvested power at the $k$-th IoT device can be given as [1], [37].

$$
E_{k}=\eta P_{0}\left|g_{d, k}+\mathbf{g}_{0} \boldsymbol{\Theta}_{0} \mathbf{g}_{r, k}\right|^{2} .
$$

where $\eta$ denotes the energy conversion efficiency, $P_{0}$ represents the transmit power of the PS. This paper assumes that each IoT device consumes all of its harvested power during the WET phase, which can maximize the achievable data rate at each IoT device during the WIT phase.

Remark 1: Several practical energy harvesting models have been investigated in [38]-[40], such as the practical energy conversion efficiency for the far-field scenario, and non-linear energy harvesting models. These models may lead to more complicated problem formulation such that it is more challenging to derive the optimal solutions of the IRS phase shifts, energy pricing, and wireless caching strategy in closed-form. In addition, the existing work studied a non-linear energy harvesting model, which is composed of a two-piece linear energy harvesting model. Specifically, the harvested power linearly improves as the received power increases and becomes saturated when the received power exceeds a certain threshold, i.e., the saturated power [41]. In this paper, it is assumed that all IoT devices adopt the first piece of this non-linear model to harvest energy without loss of generality, and this assumption typically holds during the low power transmission region, the harvested energy at the IoT devices is lower than or equal to its battery capacity. Moreover, a novel non-linear energy harvesting model will be investigated based on a fractional function, which employs a quadratic transformation (QT) to jointly optimize the IRS phase shifts and transmission time scheduling. This will be our follow-up work.

Hence, the achievable data rate at the AP from the $k$-th IoT device can be written as [1], [37]

$$
\begin{aligned}
R_{k} & =B \log \left(1+\frac{\eta P_{0}\left|g_{d, k}+\mathbf{g}_{0} \boldsymbol{\Theta}_{0} \mathbf{g}_{r, k}\right|^{2}\left|h_{d, k}+\mathbf{h}_{k} \boldsymbol{\Theta}_{k} \mathbf{h}_{r}\right|^{2}}{\sigma^{2}}\right), \\
& \forall k \in[1, K],
\end{aligned}
$$

where $B$ denotes the bandwidth of the uplink WIT, and $\sigma^{2}$ is the noise power.

\section{B. Wireless Caching Model}

In this subsection, we illustrate the wireless caching model, to be specific, the local cache of the AP is limited by $F$ which is defined as the storage limit at the AP. Also, the IoT devices transmit the IoT data as a file, the length of which is denoted by a vector $\mathbf{f}=\left[f_{1}, \ldots, f_{K}\right]$. In addition, the wireless caching strategy is represented by a binary vector $\mathbf{c}=\left[c_{1}, \ldots, c_{K}\right]^{T}$, where $c_{k} \in\{0,1\}, \forall k \in[1, K]$, denotes whether file $k$ is cached. With the request of the user, the AP first need to check its local cache and directly transmit the IoT data to the user if the requested data is cached, i.e., $c_{k}=1$, and the transmission rate is equivalent to the backhaul data rate of the $\mathrm{AP} \delta$; otherwise, this data is transmitted from the corresponding device to the user via the AP, i.e., $c_{k}=0$, and the transmission rate can be denoted by $\min \left\{R_{k}, \delta\right\}=R_{k}$ [2]. Thus, the achievable sum throughput is expressed as

$$
S=\sum_{k=1}^{K} b_{k}\left[\left(1-c_{k}\right) R_{k}+c_{k} \delta\right]
$$

where $b_{k}$ denotes the probability of the file popularity that a user requests the $k$-th file. It is assumed that the user requests these files according to their popularities, and the request probability of the user to the $k$-th file follows a Zipf distribution, e.g., $b_{k}=\frac{k^{-\epsilon}}{\sum_{i=1}^{K} i^{-\epsilon}}$, where $\epsilon$ is the skewness factor to indicate the deviation of different file popularities. On the other hand, the total cached data is required to not exceed its cache storage limit, i.e.,

$$
\sum_{k=1}^{K} f_{k} c_{k} \leq F
$$

\section{StaCkElBERG GAME Formulation}

This section considers a Stackelberg game to characterize the WET and WIT networks' strategic behaviours, aiming to maximize their utility. To be specific, we expand monetary payments by the WIT network to purchase the energy service provided by the PS. We can explain this based on a practical scenario that the WIT and WET networks may belong to different service providers. Also, the PS does not want to provide wireless charging to the IoT devices voluntarily. Hence, the AP's rewards would encourage the PS to charge the IoT devices wirelessly. In this Stackelberg game, the WIT network is considered as a leader who bids a price for the energy service released from the PS. The leader aims to maximizes its utility function which is defined as the difference between the benefits gained from the sum throughput of the data transmission plus backhaul data rate and the payment for the energy service. Thus, the leader-level problem is given by

$$
\begin{aligned}
\max _{\mathbf{c}, \boldsymbol{\Theta}, \lambda} & U_{\mathrm{WIT}}\left(P_{0}, \boldsymbol{\Theta}_{0}, \mathbf{c}, \boldsymbol{\Theta}, \lambda\right) \\
\text { s.t. } & \sum_{k=1}^{K} f_{k} c_{k} \leq F, c_{k} \in\{0,1\}, \\
& \boldsymbol{\Theta}=\left[\boldsymbol{\Theta}_{1}, \ldots, \boldsymbol{\Theta}_{K}\right], \alpha_{k, n} \in[0,2 \pi), \\
& \forall n \in[1, N], \forall k \in[1, K], \lambda \geq 0,
\end{aligned}
$$

where $U_{\mathrm{WIT}}\left(P_{0}, \boldsymbol{\Theta}_{0}, \mathbf{c}, \boldsymbol{\Theta}, \lambda\right)$ is denoted by (6) on the top of the next page, $\mu>0$ denotes the price per unit sum throughput of the WIT network, and $\lambda>0$ is the price per unit energy collection paid to the PS. The leader problem aims to optimize the wireless cache strategies, the energy pricing, as well as the phase shifts of the WIT phase. While, the WET network plays a follower's role to optimizes the transmit power of the PS and the phase shifts of the WET phase, which maximizes its utility function explained as the difference between the energy payment and the energy operation cost, which is typically defined as a quadratic function, i.e., $\mathcal{F}(x)=\tilde{\alpha} x^{2}$, where $\tilde{\alpha}>0$ 


$$
\begin{aligned}
U_{\mathrm{WIT}}\left(P_{0}, \boldsymbol{\Theta}_{0}, \mathbf{c}, \boldsymbol{\Theta}, \lambda\right) & =\mu \sum_{k=1}^{K} b_{k}\left[\left(1-c_{k}\right) B \log \left(1+\frac{\eta P_{0}\left|g_{d, k}+\mathbf{g}_{0} \boldsymbol{\Theta}_{0} \mathbf{g}_{r, k}\right|^{2}\left|h_{d, k}+\mathbf{h}_{k} \boldsymbol{\Theta}_{k} \mathbf{h}_{r}\right|^{2}}{\sigma^{2}}\right)+c_{k} \delta\right] \\
& -\lambda P_{0} \sum_{k=1}^{K}\left|g_{d, k}+\mathbf{g}_{0} \boldsymbol{\Theta}_{0} \mathbf{g}_{r, k}\right|^{2},
\end{aligned}
$$

is a pre-determined parameter [42]. Thus, the follower level problem is written as

$$
\begin{aligned}
& \max _{P_{0}, \boldsymbol{\Theta}_{0}} U_{\mathrm{WET}}\left(P_{0}, \boldsymbol{\Theta}_{0}, \lambda\right)=\lambda P_{0} \sum_{k=1}^{K}\left|g_{d, k}+\mathbf{g}_{0} \boldsymbol{\Theta}_{0} \mathbf{g}_{r, k}\right|^{2}-\mathcal{F}\left(P_{0}\right), \\
& \text { s.t. } P_{0} \geq 0, \alpha_{0, n} \in[0,2 \pi), \forall n \in[1, N] .
\end{aligned}
$$

Problem (5) and problem (7) form a Stackelberg game $\mathcal{G}$, which is modelled in the IRS assisted wireless powered caching network to exploit the strategic interaction between the WET and WIT networks. This formulated game can be defined as follows:

1) Players: the WET network, i.e., PS (follower) vs the WIT network, i.e., AP (leader).

2) Strategies:

a) Leader: wireless caching strategies $\mathbf{c}$, phase shifts of the WIT $\Theta$, and energy pricing $\lambda$.

b) Follower: transmit power of the PS $P_{0}$ and phase shifts of the WET $\boldsymbol{\Theta}_{0}$.

3) Utilities: $U_{\mathrm{WIT}}\left(P_{0}, \mathbf{c}, \boldsymbol{\Theta}, \lambda\right)$ of the WIT network and $U_{\mathrm{WET}}\left(P_{0}, \boldsymbol{\Theta}_{0}, \lambda\right)$ of the WET network.

We evaluate the optimal solution of this Stackelberg game gained by exploiting the Stackelberg equilibrium, in which both WET and WIT networks reach a consensus on the energy service, the wireless caching strategies, the phase shifts of the WET and WIT phases, as well as energy pricing. The deviation of either the WET network or the WIT network from the equilibrium will induce a performance loss. Thus, the Stackelberg equilibrium is defined as

Definition 1: Assuming that $\mathbf{c}^{*}=\left[c_{1}^{*}, \ldots, c_{K}^{*}\right], \boldsymbol{\Theta}^{*}=$ $\left[\Theta_{1}^{*}, \ldots, \Theta_{K}^{*}\right]$ and $\lambda$ are the optimal solutions of problem (5), also, $P_{0}^{*}$ and $\Theta_{0}^{*}$ are the optimal solutions of problem (7). Then, $\left(P_{0}^{*}, \boldsymbol{\Theta}_{0}^{*}, \mathbf{c}^{*}, \Theta^{*}, \lambda^{*}\right)$ satisfies the Stackelberg equilibrium of the game $\mathcal{G}$ if and only if

$$
\begin{aligned}
& U_{\mathrm{WIT}}\left(P_{0}^{*}, \boldsymbol{\Theta}_{0}^{*}, \mathbf{c}^{*}, \boldsymbol{\Theta}^{*}, \lambda^{*}\right) \geq U_{\mathrm{WIT}}\left(P_{0}^{*}, \boldsymbol{\Theta}_{0}^{*}, \mathbf{c}, \boldsymbol{\Theta}, \lambda\right) \\
& U_{\mathrm{WET}}\left(P_{0}^{*}, \boldsymbol{\Theta}_{0}^{*}, \lambda^{*}\right) \geq U_{\mathrm{WET}}\left(P_{0}, \boldsymbol{\Theta}_{0}, \lambda^{*}\right)
\end{aligned}
$$

\section{Optimal Solution of Stackelberg Game $\mathcal{G}$}

This section aims to obtain the Stackelberg equilibrium of the formulated game $\mathcal{G}$, where the best responses (optimal solutions) of the follower and the leader can be achieved by solving the follower level problem (7) and the leader level problem (5), respectively. To specific, the proposed scheme aims to achieve the optimal solution of the IRS phase shifts, the wireless caching strategy, and the energy pricing. Actually, one purpose of wireless caching technique would reduce latency. However, considering the latency minimization would require another problem formulation, which may result in the numerical optimization solution and is still time-consuming. In our work, the proposed algorithm is designed to analyze the network performance rather than make it directly applicable for the real-time applications.

\section{A. Optimal Strategy of Follower Level Problem (7)}

To solve the follower level problem (7), we first derive the optimal phase shifts of the WET in closed-form. For given $P_{0}$, solving (7) to obtain the optimal phase shifts of the WET phase is equivalent to solve the following problem

$$
\begin{aligned}
& \max _{\boldsymbol{\Theta}_{0}} \sum_{k=1}^{K}\left|g_{d, k}+\mathbf{g}_{0} \boldsymbol{\Theta}_{0} \mathbf{g}_{r, k}\right|^{2} \\
& \text { s.t. } \alpha_{k, n} \in[0,2 \pi), \forall n \in[1, N],
\end{aligned}
$$

Problem (9) is not convex due to the its non-convex unitmodulus constraint, which cannot be directly solved. To deal with it, its objective function is equivalently modified as

$$
\sum_{k=1}^{K}\left|g_{d, k}+\mathbf{g}_{0} \boldsymbol{\Theta}_{0} \mathbf{g}_{r, k}\right|^{2}=\boldsymbol{\theta}_{0} \boldsymbol{\Phi}_{1} \boldsymbol{\theta}_{0}^{H}+2 \mathcal{R}\left\{\boldsymbol{\theta}_{0} \boldsymbol{\gamma}\right\}+d_{1},
$$

where $\boldsymbol{\theta}_{0}=\left[\exp \left(j \alpha_{0,1}\right), \ldots, \exp \left(j \alpha_{0, N}\right)\right], \mathbf{a}_{k}=\operatorname{diag}\left(\mathbf{g}_{0}\right) \mathbf{g}_{r, k}$, $\boldsymbol{\Phi}_{1}=\sum_{k=1}^{K} \mathbf{a}_{k} \mathbf{a}_{k}^{H}, \gamma=\sum_{k=1}^{K} \operatorname{conj}\left(g_{d, k}\right) \mathbf{a}_{k}$, and $d_{1}=$ $\sum_{k=1}^{K} g_{d, k} \operatorname{conj}\left(g_{d, k}\right)$. By substituting (10) into problem (9) and applying a few of mathematical manipulations, we have

$$
\begin{aligned}
& \min _{\boldsymbol{\theta}_{0}} \boldsymbol{\theta}_{0} \boldsymbol{\Phi} \boldsymbol{\theta}_{0}^{H}-2 \mathcal{R}\left\{\boldsymbol{\theta}_{0} \boldsymbol{\gamma}\right\}+d \\
& \text { s.t. }\left|\boldsymbol{\theta}_{0}(n)\right|=1, \quad \forall n \in[1, N],
\end{aligned}
$$

where $\boldsymbol{\Phi}=-\boldsymbol{\Phi}_{1}, d=d_{1}$. To solve problem (11), we propose two different approaches, i.e., the Majorization-Minimization (MM) algorithm and the complex circle manifold (CCM) methods. These two algorithms have been investigated in the IRS assisted wireless networks, such as the multicell MIMO networks, the MIMO SWIPT system, and wireless powered sensor networks (WPSN) [21], [24], [37], [43].

1) Majorization-Minimization (MM) Algorithm: We first consider the MM algorithm to derive the optimal phase shifts of the WET, where the objective function and the constraint set of (11) are approximated with a sequence of tractable subproblems which are iteratively resolved [44].

Proposition 1: [44], [45] For given $\boldsymbol{\theta}^{(\boldsymbol{m})}$ and any feasible $\boldsymbol{\theta}$, 11a can be approximated at the $m$-th iteration as follows:

$$
\begin{aligned}
& f\left(\boldsymbol{\theta}_{0}\right) \triangleq \boldsymbol{\theta}_{0} \boldsymbol{\Phi} \boldsymbol{\theta}_{0}^{H}-2 \Re\left\{\boldsymbol{\theta}_{0} \boldsymbol{\gamma}\right\}+d \\
& \leq \boldsymbol{\theta}_{0} \boldsymbol{\Upsilon} \boldsymbol{\theta}_{0}^{H}-2 \Re\left\{\boldsymbol{\theta}_{0}\left[(\boldsymbol{\Upsilon}-\boldsymbol{\Phi}) \tilde{\boldsymbol{\theta}}_{0}^{H}+\boldsymbol{\gamma}\right]\right\} \\
& \quad+\tilde{\boldsymbol{\theta}}_{0}(\boldsymbol{\Upsilon}-\boldsymbol{\Phi}) \tilde{\boldsymbol{\theta}}_{0}^{H}+d \\
& =\lambda_{\max }(\boldsymbol{\Phi})\left\|\boldsymbol{\theta}_{0}\right\|^{2}-2 \mathcal{R}\left\{\boldsymbol{\theta}_{0}\left[\left(\lambda_{\max }(\boldsymbol{\Phi}) \mathbf{I}_{N \times N}-\boldsymbol{\Phi}\right) \tilde{\boldsymbol{\theta}}_{0}^{H}+\boldsymbol{\gamma}\right]\right\} \\
& \quad+\tilde{d} \triangleq g\left(\boldsymbol{\theta}_{0} \mid \tilde{\boldsymbol{\theta}}_{0}\right),
\end{aligned}
$$


where $\tilde{d}=\tilde{\boldsymbol{\theta}}_{0}\left[\lambda_{\max }(\boldsymbol{\Phi}) \mathbf{I}_{N \times N}-\boldsymbol{\Phi}\right] \tilde{\boldsymbol{\theta}}_{0}^{H}+d, \quad \boldsymbol{\Upsilon}=$ $\lambda_{\max }(\boldsymbol{\Phi}) \mathbf{I}_{N \times N}, \lambda_{\max }(\boldsymbol{\Phi})$ is the maximum eigenvalue of $\boldsymbol{\Phi}$, $\tilde{\boldsymbol{\theta}}_{0}$ denotes the approximated solution to $\boldsymbol{\theta}_{0}$ which is achieved in the previous iteration via the alternating algorithm.

By exploiting Proposition 11 we construct a surrogate function of 111a, where it can be verified that $g\left(\boldsymbol{\theta} \mid \boldsymbol{\theta}^{(m)}\right)$ in 12 satisfies all conditions in [44, Eq. 46].Thus, problem (11) is equivalently modified as

$$
\min _{\boldsymbol{\theta}_{0}} \lambda_{\max }(\boldsymbol{\Phi})\left\|\boldsymbol{\theta}_{0}\right\|^{2}-2 \mathcal{R}\left\{\boldsymbol{\theta}_{0} \tilde{\gamma}\right\} \text {, s.t. }(11 \mathrm{~b}),
$$

where $\tilde{\boldsymbol{\gamma}}=\left(\lambda_{\max }(\boldsymbol{\Phi}) \mathbf{I}_{N \times N}-\boldsymbol{\Phi}\right) \tilde{\boldsymbol{\theta}}_{0}^{H}+\boldsymbol{\gamma}$. It is apparent that $\left\|\boldsymbol{\theta}_{0}\right\|^{2}=N$ due to $\left|\boldsymbol{\theta}_{0}(n)\right|=1$. The term $\mathcal{R}\left\{\boldsymbol{\theta}_{0} \tilde{\boldsymbol{\gamma}}\right\}$ can be maximized when the phases of $\boldsymbol{\theta}_{0}(n)$ and $\tilde{\boldsymbol{\gamma}}(n)$ are identical. Hence, the optimal solution to (13) is derived as

$$
\boldsymbol{\theta}_{0}^{*}=[\exp (j \arg [\tilde{\gamma}(1)]), \ldots, \exp (j \arg [\tilde{\gamma}(N)])] .
$$

We summarize the details of the MM algorithm in Algorithm 1

Algorithm 1: The proposed MM algorithm to solve problem (11)

1) Initialization: $m$ denotes the iteration number, the accuracy $\epsilon$, the feasible solution $\tilde{\boldsymbol{\theta}}_{0}=\boldsymbol{\theta}_{0}^{(0)}$. Calculate the objective value of problem [11, i.e., $f\left(\boldsymbol{\theta}_{0}^{(1)}\right)$.

2) Repeat:

a) Calculate $\tilde{\boldsymbol{\gamma}}^{(m)}=\left(\lambda_{\max }(\boldsymbol{\Phi}) \mathbf{I}_{N \times N}-\boldsymbol{\Phi}\right)\left(\tilde{\boldsymbol{\theta}}_{0}^{(m)}\right)^{H}+\boldsymbol{\gamma}$.

b) Update $\boldsymbol{\theta}_{0}^{(m+1)}=\boldsymbol{\theta}_{0}^{*}$ in (14) and calculate $f\left(\boldsymbol{\theta}_{0}^{(m+1)}\right)$.

c) Set $m=m+1$ until convergence, i.e., $\frac{f\left(\boldsymbol{\theta}_{0}^{(m+1)}\right)-f\left(\boldsymbol{\theta}_{0}^{(m)}\right)}{f\left(\boldsymbol{\theta}_{0}^{(m+1)}\right)} \leq \epsilon$.

3) Obtain the optimal phase shifts of the WET phase, i.e., $\boldsymbol{\theta}_{0}^{*}$.

2) Complex Circle Manifold (CCM) Algorithm: Problem (11) can be solved to achieve the optimal phase shifts of the WET phase by exploiting the CCM algorithm. Its main premise is based on the derivation of a gradient descent algorithm based on the manifold space [46]. To perform this method, problem (11) is first reformulated as

$$
\begin{aligned}
& \min _{\boldsymbol{\theta}_{0}} \boldsymbol{\theta}_{0}\left(\boldsymbol{\Phi}+\kappa \mathbf{I}_{N}\right) \boldsymbol{\theta}_{0}^{H}-2 \mathcal{R}\left\{\boldsymbol{\theta}_{0} \boldsymbol{\gamma}\right\}+d, \\
& \text { s.t. }\left|\boldsymbol{\theta}_{0}(n)\right|=1, \quad \forall n \in[1, N] .
\end{aligned}
$$

Note that $\kappa>0$ in problem (15) is a positive constant to control the convergence of the CCM algorithm, and will be characterized in the following. Also, problem (11) is equivalent to (15) due to $\kappa \boldsymbol{\theta}_{0} \boldsymbol{\theta}_{0}^{H}=\kappa N$.

To proceed, we denote its objective function as $\tilde{f}\left(\boldsymbol{\theta}_{0}^{(m)}\right)$ at the $m$-th iteration. By minimizing problem $[15$, we determine the search direction, which is opposite to the gradient in Euclidean space of $\tilde{f}\left(\boldsymbol{\theta}_{0}^{(m)}\right)$, i.e.,

$$
\iota^{(m)}=-\nabla_{\boldsymbol{\theta}_{0}} \tilde{f}\left(\boldsymbol{\theta}_{0}^{(m)}\right)=-2\left(\boldsymbol{\Phi}+\kappa \mathbf{I}_{N}\right)\left(\boldsymbol{\theta}_{0}^{(m)}\right)^{H}+2 \boldsymbol{\gamma} .
$$

Next, we find the Riemannian gradient of $\tilde{f}\left(\boldsymbol{\theta}_{0}^{(m)}\right)$ at the current point $\boldsymbol{\theta}_{0}^{(m)} \in \mathcal{S}^{N}$, which is in the tangent space
$\mathcal{T}_{\boldsymbol{\theta}_{0}^{(m)}} \mathcal{S}^{N}$ 47] 3 We project the search direction $\iota^{(m)}$ in Euclidean space onto $\mathcal{T}_{\boldsymbol{\theta}_{0}^{(m)}} \mathcal{S}^{N}$, and the Riemannian gradient of $\tilde{f}\left(\boldsymbol{\theta}_{0}^{(m)}\right)$ at $\boldsymbol{\theta}_{0}^{(m)}$ is given as follows [47]:

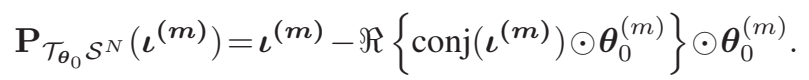

Then, we update $\boldsymbol{\theta}_{0}^{(m)}$ on the tangent space $\mathcal{T}_{\boldsymbol{\theta}_{0}^{(m)}} \mathcal{S}^{N}$, which is given as

$$
\overline{\boldsymbol{\theta}}_{0}^{(m)}=\boldsymbol{\theta}_{0}^{(m)}+\zeta \mathbf{P}_{\mathcal{T}_{\boldsymbol{\theta}_{0}} \mathcal{S}^{N}}\left(\iota^{(m)}\right),
$$

where $\zeta$ is a step size which will be characterized in the following. Finally, we map $\overline{\boldsymbol{\theta}}_{0}^{(m)}$ into the manifold $\mathcal{S}^{N}$ via retraction operation, as $\overline{\boldsymbol{\theta}}_{0}^{(m)}$ is not in $\mathcal{S}^{N}$. Note that the retraction operation is to normalize each element of $\overline{\boldsymbol{\theta}}_{0}^{(m)}$ to be unity, which is given by

$$
\boldsymbol{\theta}_{0}^{(m+1)}=\overline{\boldsymbol{\theta}}_{0}^{(m)} \odot \frac{1}{\overline{\boldsymbol{\theta}}_{0}^{(m)}} .
$$

Additionally, the following theorem required to determine the range of parameters $\kappa$ and $\zeta$ to satisfy the convergence of the CCM algorithm.

Theorem 1: [46] Provided that the parameters $\kappa$ and $\zeta$ are determined to guarantee $\kappa \geq \frac{N}{8} \varrho_{\max }(\boldsymbol{\Phi})+\|\gamma\|_{2}$, and $0<$ $\zeta<\frac{1}{\varrho_{\max }\left(\boldsymbol{\Phi}+\kappa \mathbf{I}_{N}\right)}$, respectively, the CCM algorithm yields a non-increasing sequence until convergence.

From the above-mentioned derivations, the steps of the CCM algorithm are summarized in Algorithm 2 .

Algorithm 2: The proposed CCM algorithm to solve problem (11).

1) Initialization: $m, \epsilon$ and $\boldsymbol{\theta}_{0}^{(0)}$ denote the iteration number, the accuracy, and the feasible solution, respectively. Calculate the objective value of problem [11, i.e., $f\left(\boldsymbol{\theta}_{0}^{(1)}\right)$.

2) Repeat:

a) Calculate the search direction $\iota^{(m)}$ in (16).

b) Calculate the projection of $\iota^{(m)}$ onto the tangent space according to 177 .

c) Update the tangent space via 18 .

d) Retract $\overline{\boldsymbol{\theta}}_{0}^{(m)}$ to the manifold $\mathcal{S}^{N}$ via 19 ).

e) Set $m=m+1$ until convergence, i.e., $\frac{f\left(\boldsymbol{\theta}_{0}^{(m+1)}\right)-f\left(\boldsymbol{\theta}_{0}^{(m)}\right)}{f\left(\boldsymbol{\theta}_{0}^{(m+1)}\right)} \leq \epsilon$.

3) Obtain the optimal solution $\boldsymbol{\theta}_{0}^{*}$

3) Convergence and Computational Complexity Analysis of Proposed Algorithms: Since the optimal phase shifts of the WET phase $\boldsymbol{\theta}_{0}$ can be iteratively computed via Algorithm 1 or Algorithm 2, its convergence property is investigated in the following. The MM algorithm or the CCM algorithm shows that $f\left(\boldsymbol{\theta}_{0}\right)$ is a monotonically non-increasing function at each iteration, i.e.,

$$
f\left(\boldsymbol{\theta}_{0}^{(m+1)}\right) \leq g\left(\boldsymbol{\theta}_{0}^{(m+1)} \mid \boldsymbol{\theta}_{0}^{(m)}\right) \leq g\left(\boldsymbol{\theta}_{0}^{(m)} \mid \boldsymbol{\theta}_{0}^{(m)}\right)=f\left(\boldsymbol{\theta}_{0}^{m}\right),
$$

${ }^{3}$ The tangent space of $\mathcal{S}$ at point $y_{n}$ can be defined as $\mathcal{T}_{y_{m}} \mathcal{S}^{N}=$ $\left\{z \in \mathbb{C}: \Re\left\{\operatorname{conj}(z) y_{m}\right\}=0\right\}$. Accordingly, the tangent space $\mathcal{T}_{\mathbf{y}} \mathcal{S}^{N}$ is the product of these $N$ tangent spaces $\mathcal{T}_{y_{m}} \mathcal{S}$, i.e., $\mathcal{T}_{\mathbf{y}} \mathcal{S}^{N}=\mathcal{T}_{y_{1}} \mathcal{S} \times$ $\mathcal{T}_{y_{2}} \mathcal{S} \cdots \mathcal{T}_{y_{N}} \mathcal{S}$. 
where $\boldsymbol{\theta}_{0}^{(m)}$ denotes the point generated at the $m$-th iteration. From (20), the first inequality and the third equality follow the relations of [44, Eq. 46], respectively. Also the second inequality holds via solving

$$
\boldsymbol{\theta}_{0}^{*}=\arg \min _{\boldsymbol{\theta}_{0}(n)} g\left(\boldsymbol{\theta}_{0} \mid \tilde{\boldsymbol{\theta}}_{0}\right) \text {, s.t. } \underline{11 \mathrm{~b})} \text {. }
$$

The monotonicity of the objective function in the above problem confirms that the MM algorithm or the CCM algorithm converges to a stationary point in practice due to the unitmodulus equality constraint $11 \mathrm{~b}$.

Next, we characterize the computational complexity of the proposed MM and CCM algorithms in solving problem (11). To proceed, we denote the number of iterations to achieve the convergence of the proposed MM and CCM algorithms as $I_{M M}$ and $I_{C C M}$, respectively. Both algorithms start with largest eigenvalue calculations from (12) in the MM algorithm and from Theorem 1 in the CCM algorithm, respectively, and this computational complexity of this step is given as $\mathcal{O}\left(N^{3}\right)$. Thus, the total computational complexity of the MM and $\mathrm{CCM}$ algorithms are calculated by $\mathcal{O}\left(N^{3}+I_{M M} N^{2}\right)$ and $\mathcal{O}\left(N^{3}+I_{C C M} N^{2}\right)$, which mainly depends on the number of iterations for convergence. According to the numerical evaluations [21], the MM algorithm can achieve the convergence with less number of iterations than the CCM algorithm, which implies that the MM algorithm has a lower computational complexity than the CCM counterpart.

Remark 2: In (14), we obtain the optimal phase shift of the WET phase via the proposed MM or CCM algorithm, which can independently deploy the IRS to maximize the energy signal strength at the IoT devices. Although problem (11) can be easily relaxed as a SDP, (14) brings an optimal solution which is more efficient for implementation and significantly reduces the computational complexity introduced by the SDP, especially for a large number of reflecting elements $N$.

After obtaining the optimal phase shifts at the WET phase, we denote $t=\sum_{k=1}^{K} t_{k}$, where $t_{k}=\left|g_{d, k}+\mathbf{g}_{0} \boldsymbol{\Theta}_{0}^{*} \mathbf{g}_{r, k}\right|^{2}$, $\boldsymbol{\Theta}_{0}^{*}$ can be achieved from $\boldsymbol{\theta}_{0}^{*}$. Thus, the follower level problem (7) is rewritten as

$$
\max _{P_{0}} U_{\mathrm{WET}}\left(P_{0}, \Theta_{0}^{*}, \lambda\right)=\lambda P_{0} t-\tilde{\alpha} P_{0}^{2} \text {, s.t. } P_{0} \geq 0 .
$$

To proceed, we determine the optimal strategies of the follower-level problem in terms of the transmit power of the PS (i.e., $P_{0}$ ) for given energy price $\lambda$. The following lemma is required:

Lemma 1: For given the energy prince $\lambda$, the optimal transmit power of the PS $P_{0}$ is given by $P_{0}^{*}=\frac{\lambda t}{2 \tilde{\alpha}}$.

Proof: It is easily proved that (22) is a convex problem in terms of $P_{0}$ for given energy price $\lambda$, since its objective function is a quadratic, and its constraint is affine. Thus, we directly take the first derivative of $U_{\mathrm{WET}}$ in terms of $P_{0}$ and set it to zero as $\frac{\partial U_{\mathrm{WET}}}{\partial P_{0}}=0$, which achieves the optimal solution of $P_{0}$. We thus completed the proof of Lemma 1 .

\section{B. Optimal Strategy of Leader Level Problem (5)}

In this subsection, we characterize the optimal strategy of the leader level problem (5), which is first written by replacing
$\Theta_{0}$ and $P_{0}$ with $\Theta_{0}^{*}$ and $P_{0}^{*}$, respectively, as follows:

$$
\begin{aligned}
\max _{\mathbf{c}, \boldsymbol{\Theta}_{k}, \lambda} & U_{\mathrm{WIT}}\left(P_{0}^{*}, \boldsymbol{\Theta}_{0}^{*}, \mathbf{c}, \boldsymbol{\Theta}, \lambda\right) \\
\text { s.t. } & \sum_{k=1}^{K} f_{k} c_{k} \leq F, c_{k} \in\{0,1\}, \\
& \left|\exp \left(j \alpha_{k, n}\right)\right|=1, \forall n \in[1, N], \forall k \in[1, K], \\
& \lambda \geq 0,
\end{aligned}
$$

where $U_{\mathrm{WIT}}\left(P_{0}^{*}, \boldsymbol{\Theta}_{0}^{*}, \mathbf{c}, \boldsymbol{\Theta}, \lambda\right)$ is denoted by (24) on the top of the next page. Problem (23) is not jointly convex with respect to the caching strategy $\mathbf{c}$, the phase shifts of the WIT phase $\Theta_{k}$, as well as the energy price $\lambda$. Thus, it is intractable to simultaneously find the optimal solutions of $\mathbf{c}, \boldsymbol{\Theta}_{k}$, and $\lambda$ due to the multiple coupled variables of the objective function in (23). To deal with this non-convex issue, we consider a twostep approach to optimally solve this problem. Particularly, we first derive the phase shifts of the WIT phase in a closedform. Subsequently, an AO algorithm is proposed to design the caching strategy and the energy price alternatively.

1) Optimal Phase Shifts of WIT Phase: To proceed, we first handle the phase shifts of the WIT phase $\boldsymbol{\Theta}_{k}, \forall k \in[1, K]$. The following lemma is presented to derive the optimal phase shifts of the WIT, i.e., $\boldsymbol{\Theta}_{k}$ for $\forall k \in[1, K]$.

Lemma 2: In order to achieve the optimal phase shift matrix $\Theta_{k}$, the following problem is investigated

$$
\begin{aligned}
& \max _{\boldsymbol{\Theta}_{k}}\left|\mathbf{h}_{k} \boldsymbol{\Theta}_{k} \mathbf{h}_{r}+h_{d, k}\right|^{2} \\
& \text { s.t. }\left|\exp \left(j \alpha_{k, n}\right)\right|=1, \forall n \in[1, N], \forall k \in[1, K] .
\end{aligned}
$$

Proof: From (23), it can be shown that $U_{\mathrm{WIT}}$ is a monotonically increasing function in terms of $\left|\mathbf{h}_{k} \boldsymbol{\Theta}_{k} \mathbf{h}_{r}+h_{d, k}\right|^{2}$. It then follows that solving problem (23) is equivalent to the maximization of $\left|\mathbf{h}_{k} \boldsymbol{\Theta}_{k} \mathbf{h}_{r}+h_{d, k}\right|^{2}$ for $\forall k \in[1, K]$ to obtain the optimal $\Theta_{k}$. Thus, problem (25) can be solved for given $\mathrm{w}, \tau$, and $\Theta_{0}$ to maximize the objective function (23), which completes the proof of Lemma 2 .

To solve 25], we first modify its objective function as

$$
\left|\mathbf{h}_{k} \boldsymbol{\Theta}_{k} \mathbf{h}_{r}+h_{d, k}\right|^{2}=\left|\boldsymbol{\theta}_{k} \mathbf{b}_{k}+h_{d, k}\right|^{2}, \forall k \in[1, K],
$$

where where $\mathbf{b}_{k}=\operatorname{diag}\left(\mathbf{h}_{k}\right) \mathbf{h}_{r}, \boldsymbol{\theta}_{k}=\left[\theta_{k, 1}, \ldots, \theta_{k, N_{R}}\right]=$ $\left[\exp \left(j \alpha_{k, 1}\right), \ldots, \exp \left(j \alpha_{k, N_{R}}\right)\right],\left|\theta_{k, n}\right|=1$. Via (26) and the triangle inequality,

$\left|\boldsymbol{\theta}_{k} \mathbf{b}_{k}+h_{d, k}\right| \leq \sum_{n=1}^{N}\left|\theta_{k, n} \mathbf{b}_{k}[n]\right|+\left|h_{d, k}\right|=\sum_{n=1}^{N}\left|\mathbf{b}_{k}[n]\right|+\left|h_{d, k}\right|$,

where $\mathbf{b}_{k}[n]$ is the $n$-th element of $\mathbf{b}_{k}$ and the equality holds with $\left|\theta_{k, n}\right|=1$ for $n \in[1, N]$. The optimal phase shifts can be obtained via the upper bound in 27) as

$$
\theta_{k, n}^{*}=\exp \left(j \alpha_{k, n}^{*}\right)
$$

where $\alpha_{k, n}^{*}=\arg \left(h_{d, k}\right)-\arg \left(\mathbf{b}_{k}[n]\right), \arg (\cdot)$ is the the phase operator. The optimal solution to problem (25) can be denoted by $\boldsymbol{\theta}_{k}^{*}=\left[\theta_{k, 1}, \ldots, \theta_{k, N}\right]$, and the optimal phase shift matrix $\boldsymbol{\Theta}_{k}^{*}$ is formed from $\boldsymbol{\theta}_{k}^{*}$. To proceed, we have the following proposition to characterize the beneficial role of the IRS.

Proposition 2: The optimal phase shifts of the WIT phase, i.e., $\boldsymbol{\Theta}_{k}^{*}$, can align the cascaded link (i.e., from the $k$-th IoT device to the AP via the IRS) with the direct link (i.e., from 


$$
U_{\mathrm{WIT}}\left(P_{0}^{*}, \boldsymbol{\Theta}_{0}^{*}, \mathbf{c}, \boldsymbol{\Theta}, \lambda\right)=\mu \sum_{k=1}^{K} b_{k}\left[\left(1-c_{k}\right) B \log \left(1+\frac{\eta\left(\frac{\lambda t}{2 \tilde{\alpha}}\right) t_{k}\left|h_{d, k}+\mathbf{h}_{k} \boldsymbol{\Theta}_{k} \mathbf{h}_{r}\right|^{2}}{\sigma^{2}}\right)+c_{k} \delta\right]-\lambda\left(\frac{\lambda t}{2 \tilde{\alpha}}\right) t
$$

the $k$-th IoT device to the AP), which is mathematically given by

$$
\mathbf{h}_{k} \Theta_{k}^{*} \mathbf{h}_{r}=\xi_{k} h_{d, k}, \forall k \in[1, K],
$$

where $\xi_{k}$ is a positive constant, denoting the signal strength metric of the cascaded link.

Proof: With some mathematical manipulations, the term $\left|\mathbf{h}_{k} \boldsymbol{\Theta}_{k} \mathbf{h}_{r}+h_{d, k}\right|^{2}$ is further equivalent to

$$
\begin{aligned}
& \left|\mathbf{h}_{k} \boldsymbol{\Theta}_{k} \mathbf{h}_{r}+h_{d, k}\right|^{2}=\left|\mathbf{h}_{k} \boldsymbol{\Theta}_{k} \mathbf{h}_{r}\right|^{2}+\left|h_{d, k}\right|^{2} \\
& \quad+2\left|\mathbf{h}_{k} \boldsymbol{\Theta}_{k} \mathbf{h}_{r}\right|\left|h_{d, k}\right| \cos \left[\arg \left(h_{d, k}\right)-\arg \left(\mathbf{h}_{k} \boldsymbol{\Theta}_{k} \mathbf{h}_{r}\right)\right] .
\end{aligned}
$$

From (30), it is shown that $\left|\mathbf{h}_{k} \boldsymbol{\Theta}_{k} \mathbf{h}_{r}+h_{d, k}\right|^{2}$ attains its maximum value if $\cos \left[\arg \left(h_{d, k}\right)-\arg \left(\mathbf{h}_{k} \boldsymbol{\Theta}_{k} \mathbf{h}_{r}\right)\right]=1$. Hence, the phases of both the direct and cascaded links are same, i.e., $\arg \left(h_{d, k}\right)=\arg \left(\mathbf{h}_{k} \boldsymbol{\Theta}_{k} \mathbf{h}_{r}\right)$, thus, 29) holds. This completes the proof of Proposition 2

Next, we quantify the signal strength introduced by information reflection for each IoT device.

Remark 3: By exploiting Proposition 2 for given $\tau$ and $\Theta_{0}$, the IRS can improve signal strength of the $k$-th IoT device at the AP by at most $\left(1+\xi_{k}\right)^{2}$ times compared with the case without IRS. In addition, $\xi_{k}$ for $\forall k \in[1, K]$ is proportional to the number of the IRS reflecting elements. Hence, a larger number of the reflecting elements will lead to a more significant performance gains for the utility of the AP. Moreover, the IRS deployment as well as path loss model of the cascaded link also have a significant impact on the received signal strength [48].

2) Optimal Solutions of Caching Strategy and Energy Price: In Section IV-B1 the optimal phase shifts of the WIT phase is derived by $\boldsymbol{\theta}_{k}^{*}$ in (28), and equivalently the optimal solution (i.e., $\boldsymbol{\Theta}_{k}^{*}$ ) is obtained from $\boldsymbol{\theta}_{k}^{*}$. Let us denote $\tilde{t}_{k}=\left|h_{d, k}+\mathbf{h}_{k} \boldsymbol{\Theta}_{k}^{*} \mathbf{h}_{r}\right|^{2}$, problem (23) is written as

$$
\begin{aligned}
& \max _{\mathbf{c}, \lambda} U_{\mathrm{WIT}}\left(P_{0}^{*}, \boldsymbol{\Theta}_{0}^{*}, \mathbf{c}, \boldsymbol{\Theta}^{*}, \lambda\right) \\
&= \mu \sum_{k=1}^{K} b_{k}\left[\left(1-c_{k}\right) B \log \left(1+\frac{\eta\left(\frac{\lambda t-\beta}{2 \alpha}\right) t_{k} \tilde{t}_{k}}{\sigma^{2}}\right)+c_{k} \delta\right] \\
& \quad-\frac{\lambda^{2} t^{2}-\beta \lambda t}{2 \alpha}, \\
& \text { s.t. } \sum_{k=1}^{K} f_{k} c_{k} \leq F, c_{k} \in\{0,1\}, \\
& \lambda \geq 0 .
\end{aligned}
$$

Problem (31) is jointly non-convex due to the coupling of the Boolean variables $\mathbf{c}$ and the logarithm function with respect to the energy price $\lambda$. To work around the non-convex problem, we propose an $\mathrm{AO}$ algorithm to design the caching strategy/energy price for given energy price/caching strategy.

1) Optimize caching strategy for given energy price: We fix the energy price to design the caching strategy, thus, problem (31) can be rewritten as

$$
\begin{aligned}
& \max _{\mathbf{c}} \mu \sum_{k=1}^{K} b_{k} c_{k}\left(\delta-R_{k}\right)+\mu \sum_{k=1}^{K} b_{k} R_{k}-\frac{\lambda^{2} t^{2}}{2 \alpha} \\
& \text { s.t. } \sum_{k=1}^{K} f_{k} c_{k} \leq F, \quad c_{k} \in\{0,1\},
\end{aligned}
$$

Problem (32) can be regarded as a $0-1$ knapsack problem, which can be resolved by various techniques such as approximation algorithm and dynamic programming [2], [49].

2) Optimize energy price for given caching strategy: Now, we fix the caching strategy to optimize the energy price. For convenience, two sets $\mathcal{S}^{+}=\left\{k \mid c_{k}=1, k \in[1, K]\right\}$ and $\mathcal{S}^{-}=\left\{k \mid c_{k}=0, k \in[1, K]\right\}$ are denoted as the cached and uncached files, respectively. Problem (31) is thus transformed as

$$
\begin{aligned}
\max _{\lambda} \mu \sum_{k \in \mathcal{S}^{-}} b_{k} B \log \left(1+\frac{\eta\left(\frac{\lambda t}{2 \tilde{\alpha}}\right) t_{k} \tilde{t}_{k}}{\sigma^{2}}\right) \\
+\mu \delta \sum_{k \in \mathcal{S}^{+}} b_{k}-\frac{\lambda^{2} t^{2}}{2 \alpha} \\
\text { s.t. } \lambda \geq 0 .
\end{aligned}
$$

It can be easily shown that problem (33) is convex in terms of $\lambda$. Since its objective function is composed of a sum of the logarithm functions, a constant, and a quadratic function, also, the constraint is linear function, which confirms the convexity of (33). Thus, we set the first derivative of the objective function in (33) equal to zero, which is given as

$$
\frac{\partial U_{\mathrm{WIT}}}{\partial \lambda}=\mu B \sum_{k \in \mathcal{S}^{-}} \frac{b_{k} \eta t t_{k} \tilde{t}_{k}}{2 \alpha \sigma^{2}+\eta \lambda t t_{k} \tilde{t}_{k}}-\frac{\lambda t^{2}}{2 \alpha}=0 .
$$

From (34), it is not easy to obtain the optimal energy price $\lambda$ in closed-form. To tackle this issue, we design an algorithm to iteratively update $\lambda$, which is expressed as

$$
\lambda^{(l+1)}=\frac{\alpha \mu B}{t^{2}} \sum_{k \in \mathcal{S}^{-}} \frac{b_{k} \eta t t_{k} \tilde{t}_{k}}{2 \alpha \sigma^{2}+\eta \lambda^{(l)} t t_{k} \tilde{t}_{k}},
$$

where $l$ is the number of iterations. The iterative algorithm to update $\lambda$ is summarized in Algorithm 3 , which update $\lambda$ in an iterative manner until convergence [2].

Algorithm 3: The iterative algorithm to optimize $\lambda$

1) Initialization: $l, \epsilon, \lambda^{(0)}$ denote the iteration number, the accuracy, and the feasible solution, respectively.

2) Repeat: for given $\lambda^{(l)}$ at the $l$-th iteration.

a) Obtain $\lambda^{(l+1)}$ via (35).

b) Set $l=l+1$ until convergence.

3) Output: $\lambda^{*}=\lambda^{(l+1)}$. 
C. Alternating Optimization Based IRS Assisted Wireless Powered Caching Algorithm

In this subsection, we consider an AO based IRS assisted wireless powered caching algorithm to jointly design the leader level problem (5). We first jointly design the optimal phase shifts of the WET and the optimal transmit power of the PS by solving the follower level problem (7) in Section IV-A Then, we consider the AO algorithm to optimize the caching strategy and the energy price alternatively, which follows the optimal derivation of the phase shifts of the WIT phase. Based on these manipulations, we summarize the proposed AO based IRS assisted wireless powered caching algorithm in Algorithm 4

Algorithm 4: The proposed AO based IRS assisted wireless powered caching algorithm

1) Initialization: $\mathbf{c}^{(0)}, \lambda^{(0)}, i$ and $\epsilon$ denote the feasible solutions of the caching strategy and the energy price, the iteration number as well as the accuracy, respectively. Calculate the objective value of problem (7), denoted by $U_{\mathrm{WIT}}^{(1)}$.

2) Obtain the optimal phase shifts of the WET phase $\boldsymbol{\theta}_{0}$ via Algorithm 1 or Algorithm 2 .

3) Obtain the optimal transmit power of the PS $P_{0}$ via Lemma 1

4) Obtain the optimal phase shifts of the WIT phase $\boldsymbol{\theta}_{k}, \forall k \in[1, K]$ via $[28$.

5) Repeat: the $\mathrm{AO}$ algorithm with iteration number $i$

a) Calculate the caching strategy $\mathbf{c}^{(i+1)}$ via the $0-1$ knapsack problem (32) for given energy price $\lambda^{(i)}$.

b) Calculate the energy price $\lambda^{(i+1)}$ via Algorithm 3 for given caching strategy $\mathbf{c}^{(i+1)}$.

c) Set $i=i+1$ until convergence, i.e., $\frac{U_{\mathrm{wIT}}^{(i+1)}-U_{\mathrm{wIT}}^{(i)}}{U_{\mathrm{wIT}}^{(i+1)}} \leq$ $\epsilon$.

d) AO Output: the optimal solutions of the caching strategy and the energy price, i.e., $\mathbf{c}^{*}=\mathbf{c}^{(i+1)}$ and $\lambda^{*}=\lambda^{(i+1)}$.

6) Output: $U_{\mathrm{WIT}}\left(P_{0}^{*}, \boldsymbol{\Theta}_{0}^{*}, \mathbf{c}^{*}, \boldsymbol{\Theta}^{*}, \lambda^{*}\right)$.

\section{Numerical Results}

This section demonstrates the numerical results to validate the overall performance of the proposed algorithm. We consider a three-dimensional (3-D) coordinates to illustrate the network deployment in Fig. 2, where the PS, the IRS and the AP are placed as $\left(X_{P S}, Y_{P S}, Z_{P S}\right),\left(X_{I R S}, Y_{I R S}, Z_{I R S}\right)$ and $\left(X_{A P}, Y_{A P}, Z_{A P}\right)$, while the IoT devices are randomly located within a circular area of $x-z$ coordinates centered at $(0,0)$ with radius $5 \mathrm{~m}$. Specifically, we set $X_{P S}=-10$, $X_{A P}=10, X_{I R S}=-2, Y_{I R S}=6, Y_{P S}=Z_{P S}=$ $Y_{A P}=Z_{A P}=Y_{k}=Z_{I R S}=0$. All channel coefficients are composed of distance-dependent path loss and small-scale fading, i.e., $\mathrm{g}=P_{L} \overline{\mathbf{g}}$. Note that $P_{L}=A d^{-\varepsilon}$ and $\overline{\mathrm{g}}$ denotes the path loss model and the small-scale coefficients for the corresponding channel, respectively, also, $A=-20 \mathrm{~dB}, \varepsilon$ and $d$ are the path loss exponent and the distance between

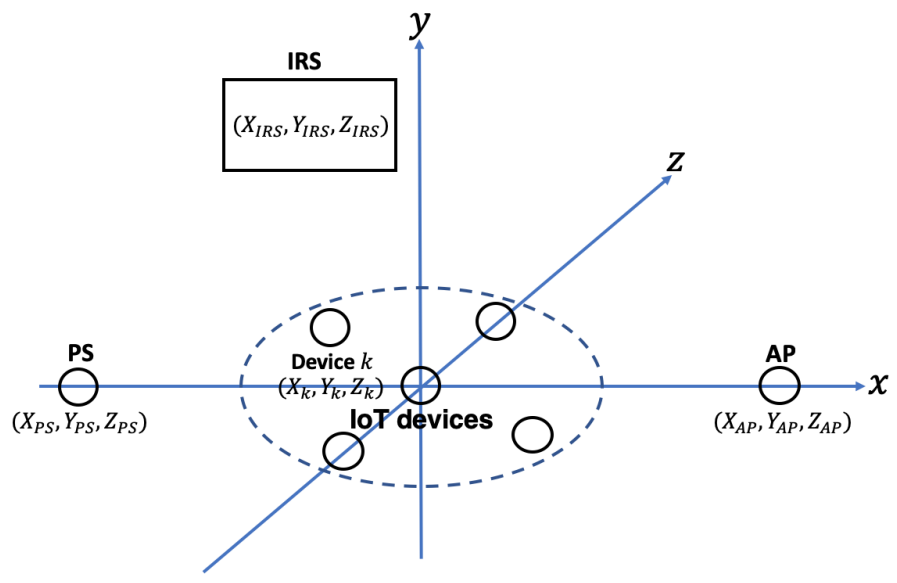

Fig. 2: System deployment.

any two nodes, respectively. The small-scale channel coefficients from the PS to the IRS, from the IRS to the $k$ th IoT device, from the $k$-th IoT device to the IRS, as well as from the IRS to the AP are modelled as Rician fading models given by $\overline{\mathrm{g}}_{0}=\sqrt{\frac{K_{1}}{K_{1}+1}} \mathbf{g}_{0}^{\mathrm{LOS}}+\sqrt{\frac{1}{K_{1}+1}} \mathbf{g}_{0}^{\mathrm{NLOS}}$, $\overline{\mathbf{g}}_{r, k}=\sqrt{\frac{K_{1}}{K_{1}+1}} \mathbf{g}_{r, k}^{\mathrm{LOS}}+\sqrt{\frac{1}{K_{1}+1}} \mathbf{g}_{r, k}^{\mathrm{NLOS}}, \overline{\mathbf{h}}_{k}=\overline{\mathbf{g}}_{r, k}^{T}$, and $\overline{\mathbf{h}}_{r}=\sqrt{\frac{K_{1}}{K_{1}+1}} \mathbf{h}_{r}^{\mathrm{LOS}}+\sqrt{\frac{1}{K_{1}+1}} \mathbf{h}_{r}^{\mathrm{NLOS}}$, where $\mathbf{g}_{0}^{\mathrm{LOS}}, \mathbf{g}_{r, k}^{\mathrm{LOS}}$, and $\mathbf{h}_{r}^{\mathrm{LOS}}$ denote the line-of-sight (LOS) deterministic components of the corresponding channel coefficients; $\mathbf{g}_{0}^{\mathrm{NLOS}}, \mathrm{g}_{r, k}^{\mathrm{NLOS}}$, and $\mathbf{h}_{r}^{\mathrm{NLOS}}$ are the non-line-of-sight (NLOS) components of the corresponding channel coefficients which follow the Rayleigh fading; $K_{1}$ is the Rician factor which is set to $5 \mathrm{~dB}$ for convenience and without loss of generality. The remaining small-scale channel coefficients are generated as the Gaussian random variable, i.e., $\mathcal{C N}(0,1)$. Unless otherwise specified, the configurations of the numerical evaluations are summarized as: the number of IoT devices $K=10$, the number of IRS reflecting elements $N=70$, the system bandwidth $B=180 \mathrm{kHz}$, the noise power density is $-174 \mathrm{dBm} / \mathrm{Hz}$, the energy conversion efficiency $\eta=0.8$, the energy cost coefficient $\alpha=1$, the backhaul data rate of AP $\delta=2 \mathrm{Mb} / \mathrm{s}$, the cache storage limit $F=2 \mathrm{Mb}$, and the Zipf file popularity skewness factor $\epsilon=0.6$.

To highlight the overall performance of the proposed scheme, we also evaluate the performance of the following benchmark schemes under the same configurations for comparison.

1) Optimal caching strategy with random phase shifts: the phase shifts of both WET and WIT phases are uniformly and randomly distributed in $[0,2 \pi)$.

2) Optimal caching strategy without IRS: the conventional wireless powered caching system without IRS is considered.

3) Optimal caching strategy without direct link: the direct links between the PS and the IoT devices as well as the IoT devices and the AP are too weak so that they can be neglected.

4) IRS assisted wireless powered network without caching: the optimal phase shifts of the WET and WIT phases 
are designed.

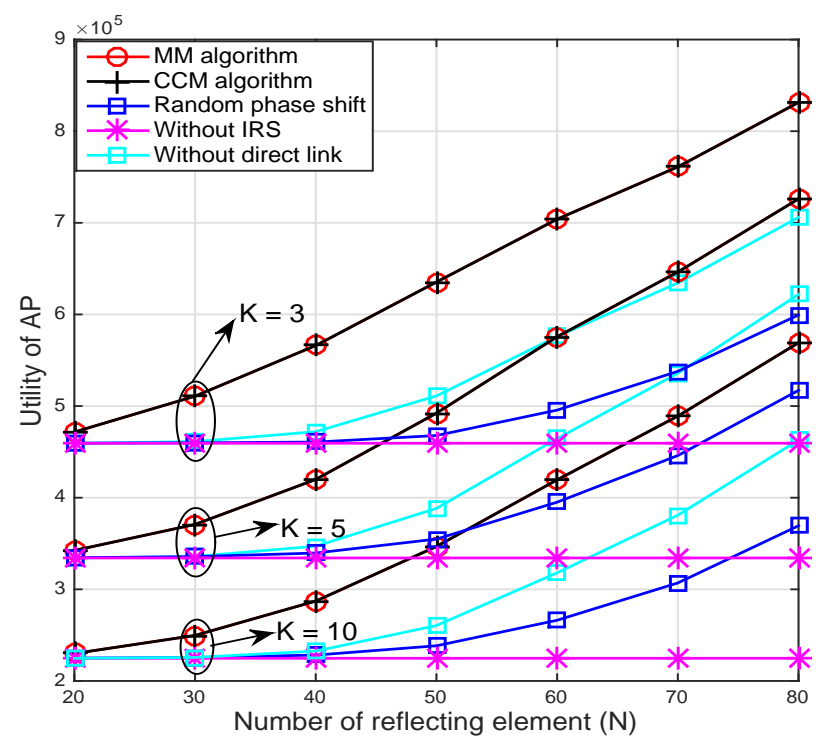

Fig. 3: The utility of AP versus number of reflecting element at the $\operatorname{IRS}(N)(\delta=2 \mathrm{Mb} / \mathrm{s}, F=2 \mathrm{Mb}$, and $\epsilon=0.6)$.

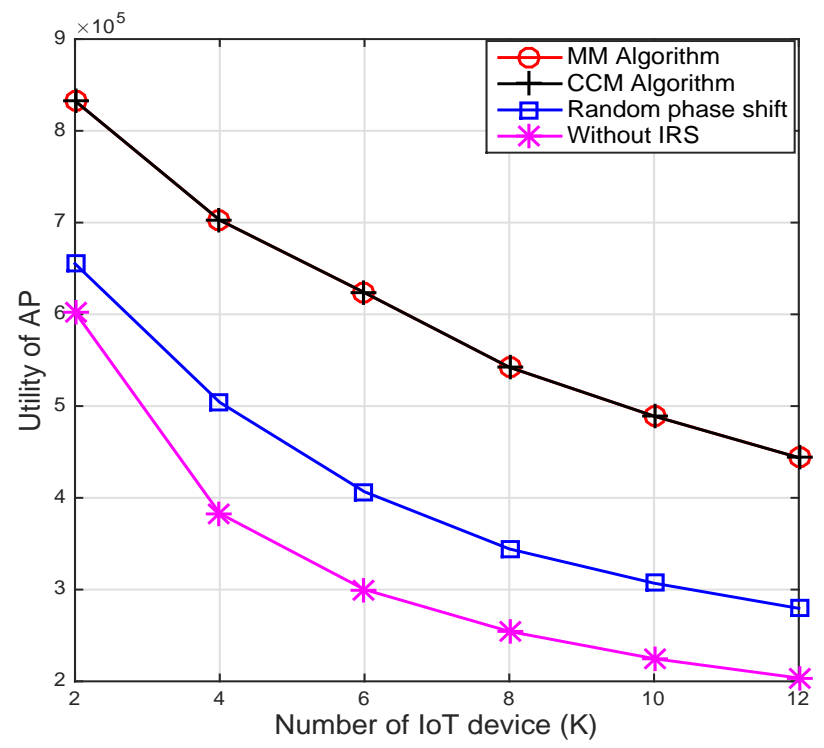

Fig. 4: The utility of AP versus number of IoT device $(K)$ $(N=70, \delta=2 \mathrm{Mb} / \mathrm{s}, F=2 \mathrm{Mb}$, and $\epsilon=0.6)$.

First, we evaluate the utility of the AP versus the number of the reflecting element $N$ at the IRS in Fig. 3 with different numbers of IoT devices ( $K=3,5,10)$, where the optimal caching strategy is applied to three different schemes (e.g. optimal phase shifts, random phase shifts, and without IRS). From this figure, one can observe that the utility of AP has an increasing trend with respect to $N$, especially in a larger number of reflecting elements region. This is expected to reveal the fact that the IRS can effectively improve the overall performance of the wireless powered caching system. Also, the proposed MM and CCM methods produce identical

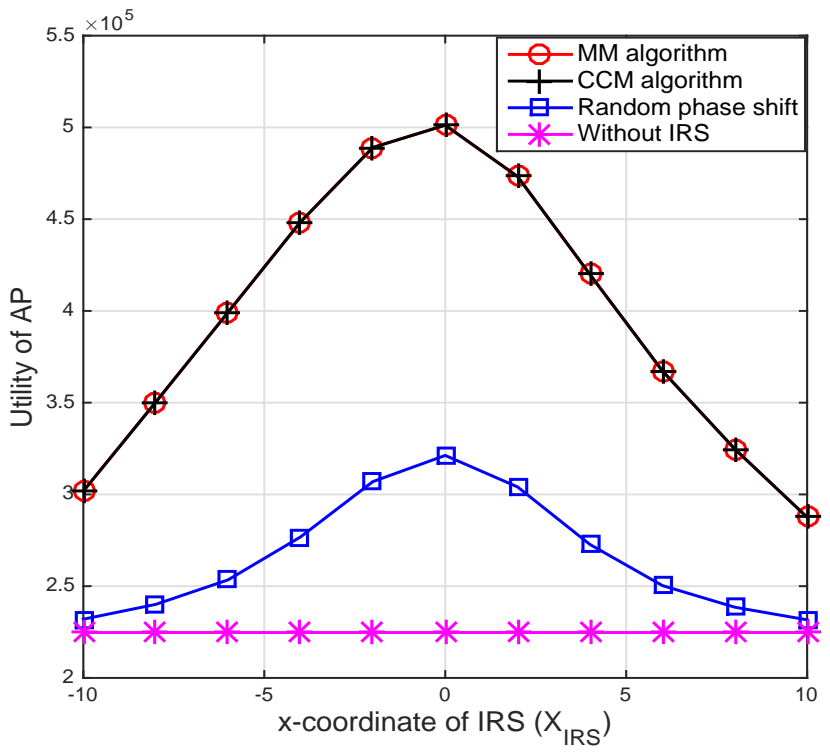

Fig. 5: The utility of AP versus $x$-coordinate of the IRS $\left(X_{I R S}\right)$ $(K=10, N=70, \delta=2 \mathrm{Mb} / \mathrm{s}, F=2 \mathrm{Mb}$, and $\epsilon=0.6)$.

results, which validates the local optimality of the IRS phase shifts and demonstrates the effectiveness of the proposed methods. Both proposed schemes significantly outperform the other two benchmark schemes, i.e., random phase shifts, and without IRS. The gap between the proposed schemes and these benchmark schemes becomes larger as $N$ increases due to a higher array gain, leading to enhanced energy/information signal reception, thus improved performance. Moreover, the proposed scheme outperforms that without the direct link, which can be explained by the fact that the proposed scheme includes both links, i.e., direct and reflection links, which produces a better performance in terms of energy harvesting and information transmission; Whereas the scheme without the direct link only has the reflection link, which relies on the IRS to reflect the energy and information during the downlink WET and uplink WIT, respectively. As such, the scheme without the direct link incurs a significantly degradation compared with the proposed scheme.

Next, Fig. 4 demonstrates the utility of AP with different numbers of IoT devices $K$. It can be observed that supporting more IoT devices would degrade the utility of the AP. This follows from the fact that files more likely not be cached at the AP with a larger $K$, and need to be delivered from the IoT devices by utilizing the harvested power, which in turn results in a lower utility of the AP.

We then evaluate the utility of the AP versus X-coordinate of the IRS $X_{I R S}$ in Fig. 5

As seen in this figure, we vary the $\mathrm{X}$-coordinate of IRS $X_{I R S}$ from -10 to 10 . When the IRS moves from -10 to 0 , the utility of the AP demonstrates an increasing behaviour, this may be explained by the fact that the higher energy reflection efficiency improves the utility function of the AP since the distance between the IoT devices and IRS becomes closer and plays a dominant role to enhance the utility of the AP. As the IRS moves from 0 to 10, longer distance 
between the PS/IoT devices and the IRS, which affects the WET and WIT simultaneously such that the utility of the AP is significantly reduced. Apparently, this trend shows that the optimal deployment of the IRS effectively improves the energy collection at the IoT devices so as to maximize the information reception at the AP.

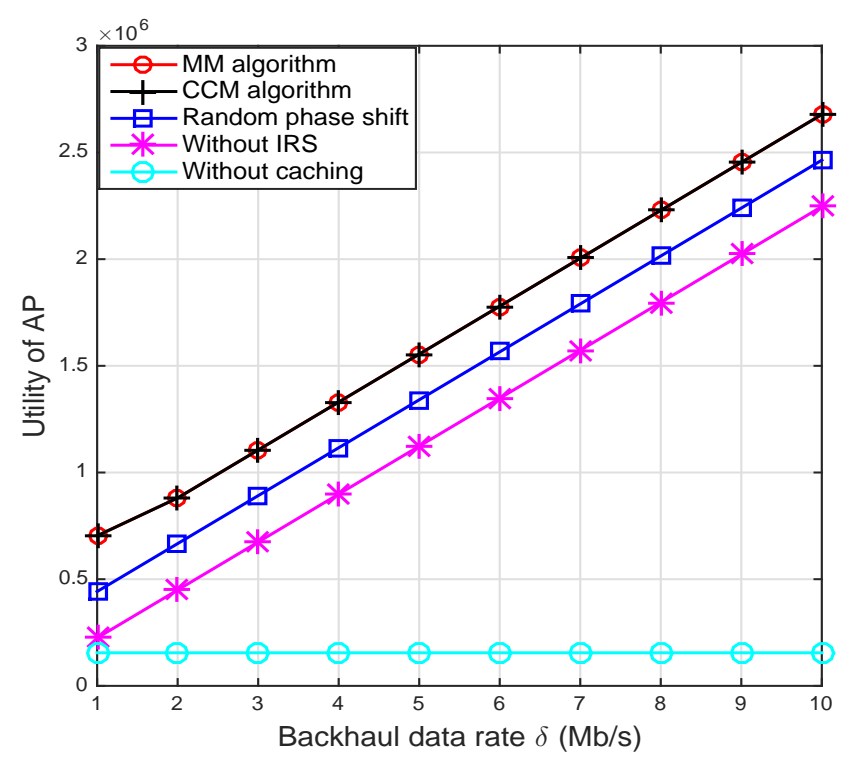

Fig. 6: The utility of AP versus backhaul data rate $(\delta)(K=10$, $N=70, F=2 \mathrm{Mb}$, and $\epsilon=0.6)$.

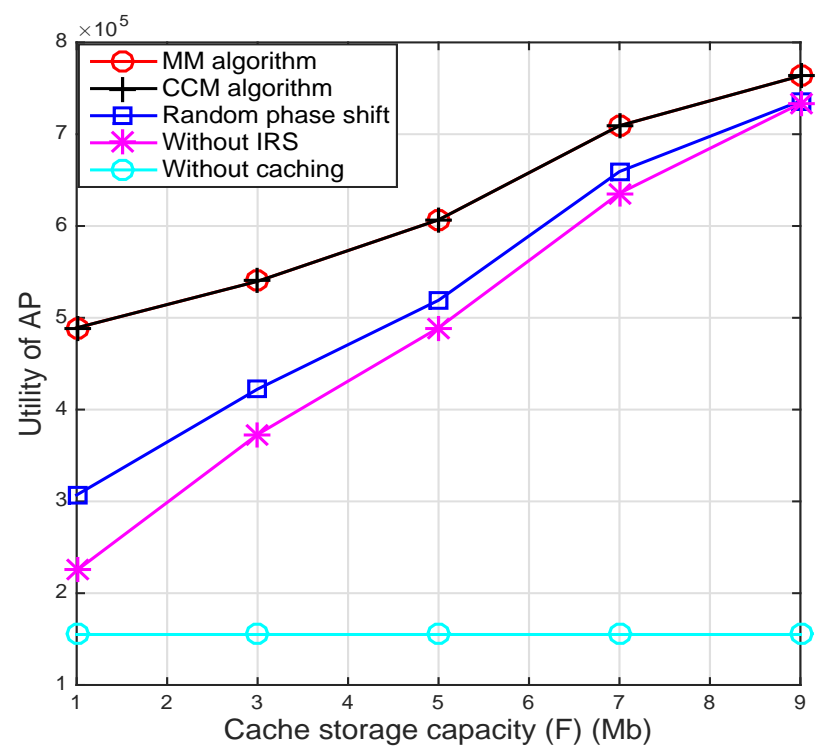

Fig. 7: The utility of AP versus cache storage limit $(F)(K=10$, $N=70, \delta=2 \mathrm{Mb} / \mathrm{s}$, and $\epsilon=0.6)$.

In Fig. 6, we demonstrate the utility of the AP versus the backhaul data rate $\delta$. It is seen from this figure that the optimal caching strategies have an increasing trend with respect to $\delta$, and outperforms the counterpart without caching, which remain stable with $\delta$. Fig. 7 demonstrates the utility of the AP versus the cache storage capacity $F$, where it is expected that

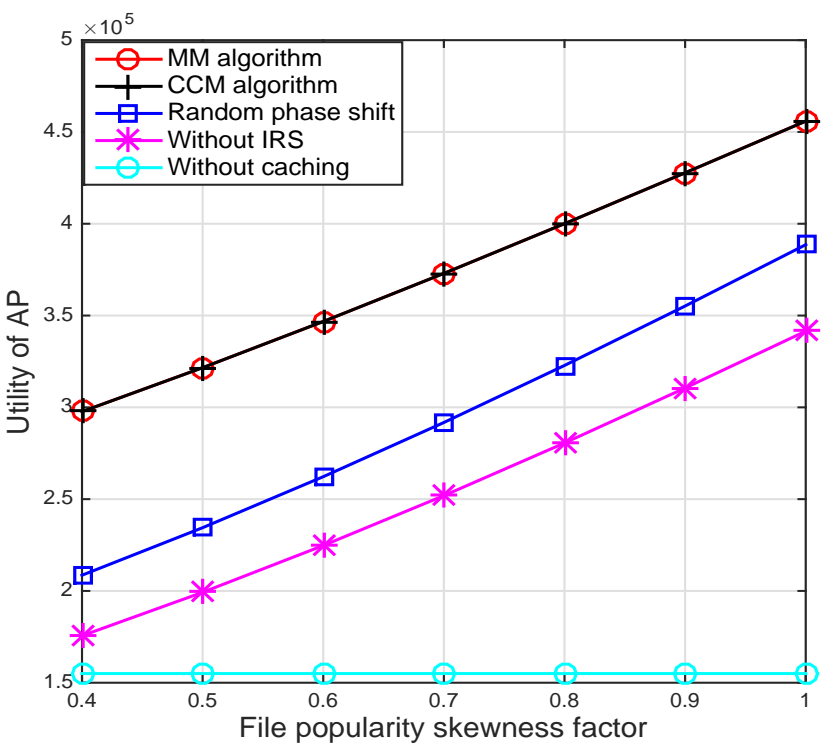

Fig. 8: The utility of AP versus file popularity skewness factor $(\epsilon)$ $(K=10, N=70, \delta=2 \mathrm{Mb} / \mathrm{s}$, and $F=2 \mathrm{Mb}$ ).

this utility increases with respect to $F$. This is due to the fact that more files may be cached with a larger cache storage limit, which leads to an increasing utility of the AP. While, the IRS assisted wireless powered network without caching remains unchanged with respect to $F$. In Fig. 8 , we evaluate the impact of file popularity skewness factor $\epsilon$ on the utility of the AP, where the optimal cache strategies increase the utility with respect to $\epsilon$. This is due to the fact that larger skewness factor leads to a larger divergence of the file popularity distribution, and more IoT devices are interested in less popular files which are cached at the AP. It can also be seen that the utility of the IRS assisted wireless powered network without caching remains unchanged with respect to $\epsilon$, since the it does not adopt cache to deliver data.

Moreover, we describe the utility of the AP versus the pathloss exponent between the PS and the IRS, the IRS and the IoT devices, as well as the IRS and the AP in Fig. 9, Fig. 10, and Fig. 11 respectively. From Fig. 9, we observe a declining trend of the utility of the AP with respect to the pathloss exponent between the PS and the IRS in the IRS assisted wireless powered network, this is expectedly since a largerscale fading between the PS and the IRS will lead to a weaker energy reception from the PS such that the beneficial role of the IRS is diminished. In addition, Fig. 10 illustrates that the utility of the AP has a significantly decreasing trend with the pathloss exponent between the IRS and the IoT devices, which can be explained by the fact that a larger-scale fading between the IRS and the IoT devices adversely affects energy reflection during the WET phase or information reception during the WIT phase. Similar trend and argument from Fig. 11 can be observed here, where a larger-scale fading between the IRS and the AP leads to a weaker channel gain, thus diminishing the information reflection of the IRS during the WIT phase.

Furthermore, we characterize the impact of the discrete phase shifts on the utility of the AP. The performance of 


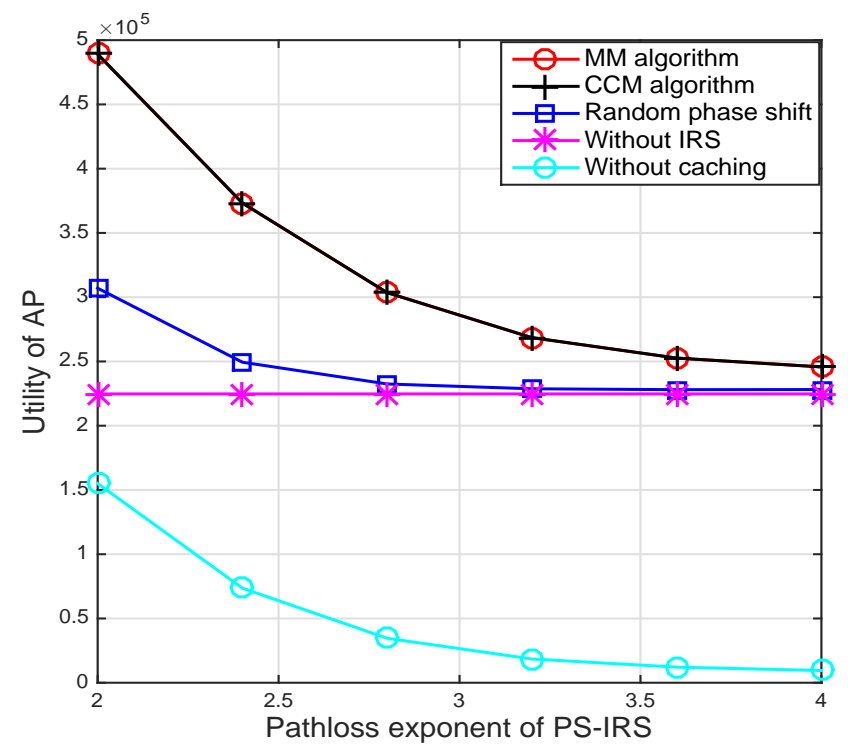

Fig. 9: The utility of AP versus pathloss exponent between the PS and the IRS $(K=10, N=70, \delta=2 \mathrm{Mb} / \mathrm{s}, F=2 \mathrm{Mb}$, and

$$
\epsilon=0.6) \text {. }
$$

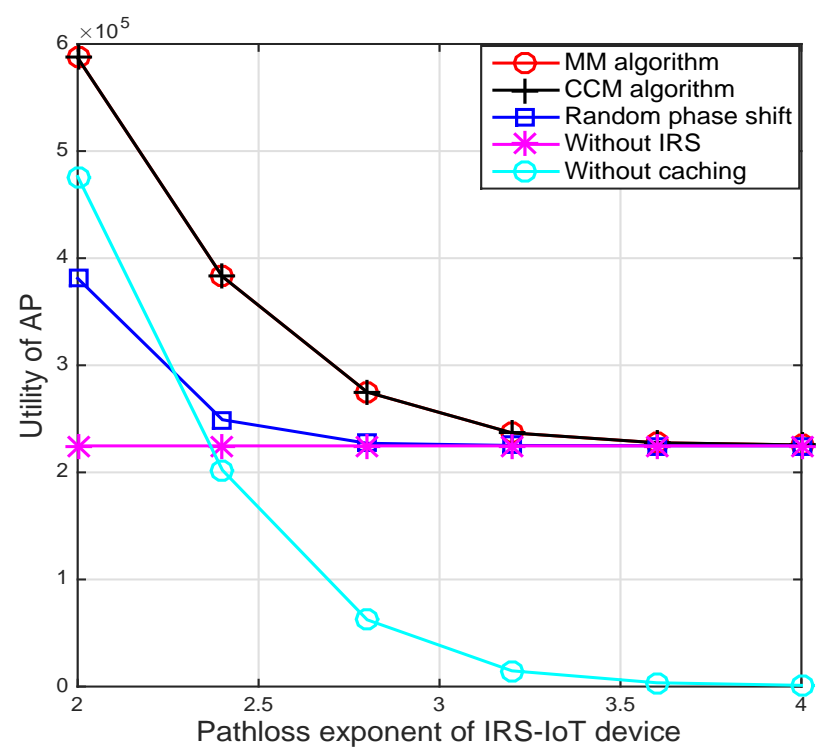

Fig. 10: The utility of AP versus pathloss exponent between the IRS and the IoT devices $(K=10, N=70, \delta=2 \mathrm{Mb} / \mathrm{s}$, $F=2 \mathrm{Mb}$, and $\epsilon=0.6)$.

continuous phase shifts is compared to that of discrete phase shifts [50] in Fig. 12, which characterizes the utility of the AP for the EBCD and CCM algorithms with the continuous and discrete phase shifts, respectively. Specifically, the performance of the continuous phase shifts represents an upper bound for its discrete counterpart in terms of the utility of the AP, and the gap between them gradually decreases as number of bits used for phase resolutions increases from 1 bit to 10 bits. This is explained by the fact that the quantized phase shifts result in an imperfect alignment such that a performance loss of energy and information receptions is

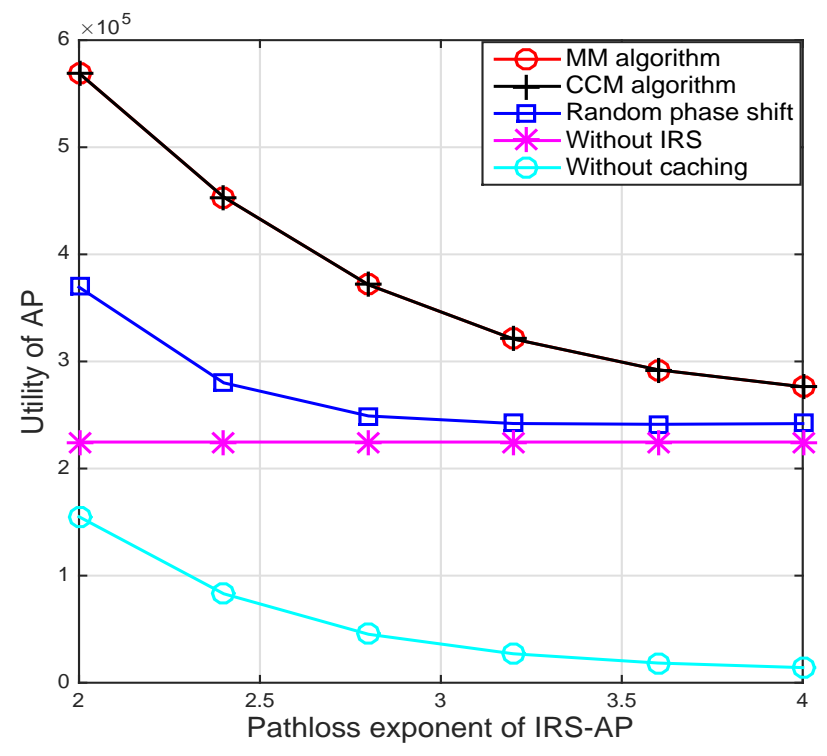

Fig. 11: The utility of AP versus pathloss exponent between the IRS and the $\operatorname{AP}(K=10, N=70, \delta=2 \mathrm{Mb} / \mathrm{s}, F=2 \mathrm{Mb}$, and $\epsilon=0.6)$.

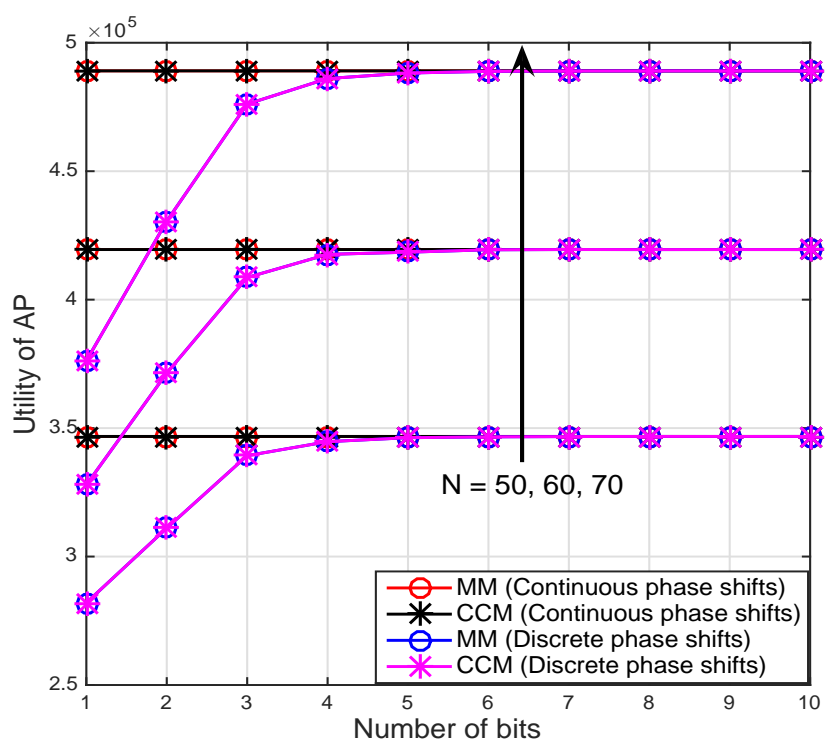

Fig. 12: The utility of AP versus number of bits $(K=10$, $\delta=2 \mathrm{Mb} / \mathrm{s}, F=2 \mathrm{Mb}$, and $\epsilon=0.6)$.

induced. In addition, a larger number of bits will yield a higher interval density of phase shifts to be selected for energy and information reflections, thus approaching the performance of the continuous counterpart in terms of the utility of the AP.

To evaluate the impact of the system deployment, Fig. 13 considers a larger deployment of physical distance, where the PS, AP, IRS are deployed as $\left(X_{P S}=20, Y_{P S}=\right.$ $\left.0, Z_{P S}=0\right),\left(X_{A P}=20, Y_{A P}=0, Z_{A P}=0\right)$, and $\left(X_{I R S}=2, Y_{I R S}=8, Z_{I R S}=0\right)$, and all IoT devices are randomly located within a circular area of $x-z$ coordinates centered at $(0,0)$ with radius $8 \mathrm{~m}$. From this result, one can observe that the proposed scheme (i.e., MM or CCM 


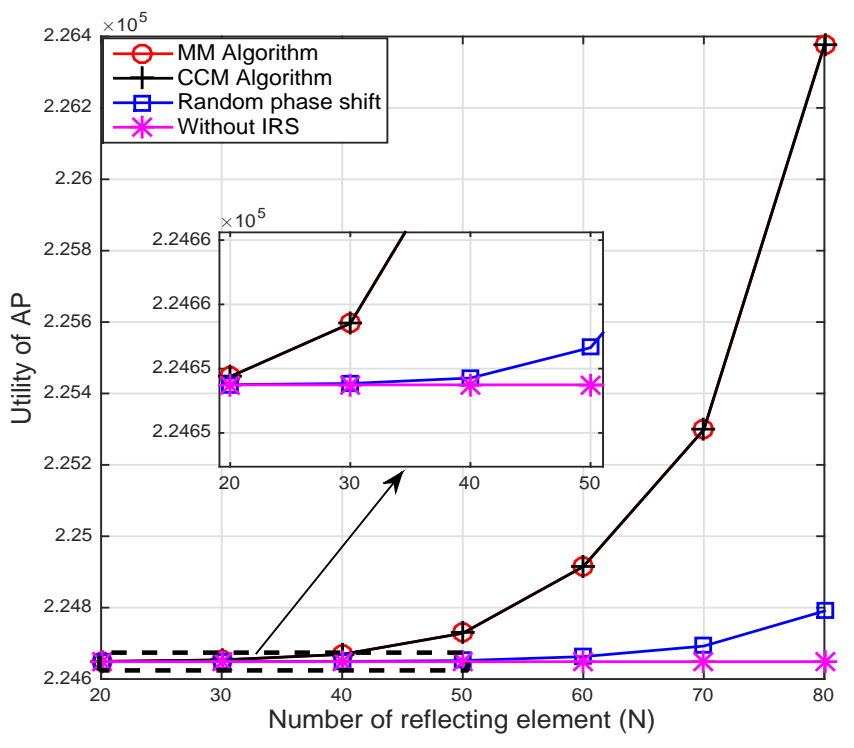

Fig. 13: The utility of AP versus number of reflecting elements $N$ $(K=10, \delta=2 \mathrm{Mb} / \mathrm{s}, F=2 \mathrm{Mb}$, and $\epsilon=0.6)$.

algorithm) shows an increasing trend with respect to $N$ and provides a better performance than the benchmark schemes, i.e., the schemes with random phase shifts and without IRS, especially for larger number of reflecting elements, which verifies the optimality of the IRS phase shifts, and highlights the benefits induced by the IRS, respectively. The scheme with the random phase shifts also highlights the benefit of using IRS compared with that without IRS after $N \geq 30$, whereas the scheme without IRS remain constant with respect to the IRS, this is due to the fact that the IRS did not participate the energy or information reflection during the downlink WET and uplink WIT. Compared to Fig. 3, this larger scenario magnifies the physical distance between each two nodes, which enlarges the propagation fading so as to significantly degrade the utility performance at the AP. Also, the larger scenario in Fig. 13 leads to a smaller increase of utility performance at the AP with respect to $N$, especially when the number of reflecting elements is small, this is due to the fact that larger wireless propagation fading or path loss can weaken the impact of the utility performance at the AP. As such, this result suggests that this wireless powered caching network should deploy larger number of reflecting elements to combat the larger-fading scenario.

\section{CONCLUSION}

In this paper, we proposed the IRS assisted wireless powered caching system, where the IoT devices collect energy from the PS to support data transmission. The AP is equipped with a wireless cache to store uploaded files from the IoT devices according to their popularity. To optimize the overall performance, we proposed a Stackelberg game to exploit the PS and AP interaction. We first solved the follower level problem, where the MM and CCM algorithms were proposed to derive the optimal phase shifts of the WET phase in closed-form, and the optimal transmit power of the PS was subsequently derived. Then, we proposed an AO algorithm to solve the leader level problem. Specifically, we derive the optimal phase shifts of the WIT phase in closed-form and design the optimum wireless caching strategies and the energy price. Finally, the numerical results validate that the proposed scheme can introduce a significant performance gain. The IRS and the wireless caching bring significant utility performance gains compared to the benchmark schemes. For the future work, we would like to investigate the IRS assisted wireless power caching systems with more multiple access schemes, such as time division multiple access (TDMA), frequency division multiple access (FDMA), non-orthogonal multiple access (NOMA). Furthermore, the IRS assisted mobile edge computing and wireless caching would be investigated for joint integration of the communication, sensing and computing. Moreover, the channel estimation of the cascaded CSI related to the IRS may be one of our future work. Specifically, robust design will be studied to deal with the imperfect channel estimation, where the cascaded channel estimation errors are modelled to be deterministically or statistically bounded. Additionally, distributed IRSs can be deployed to overcome the larger-fading scenario, since multiple IRSs can introduce a massive number of IRS reflecting elements in a distributed way to strengthen the energy reception at the IoT devices and the information reception at the AP. This will be a topic for future investigation.

\section{REFERENCES}

[1] Z. Chu, F. Zhou, Z. Zhu, R. Q. Hu, and P. Xiao, "Wireless powered sensor networks for internet of things: Maximum throughput and optimal power allocation," IEEE Internet Things J., vol. 5, no. 1, pp. 310-321, Feb. 2018

[2] J. Yao and N. Ansari, "Caching in energy harvesting aided internet of things: A game-theoretic approach," IEEE Internet Things J., vol. 6, no. 2, pp. 3194-3201, Apr. 2019.

[3] R. Zhang and C. K. Ho, "MIMO broadcasting for simultaneous wireless information and power transfer," IEEE Trans. Wireless Commun., vol. 12, pp. 1989-2001, May 2013.

[4] S. Bi, C. K. Ho, and R. Zhang, "Wireless powered communication: opportunities and challenges," IEEE Commun. Mag., vol. 53, no. 4, pp. 117-125, 2015.

[5] K. Huang and X. Zhou, "Cutting the last wires for mobile communications by microwave power transfer," IEEE Commun. Mag., vol. 53, no. 6, pp. 86-93, Jun. 2015.

[6] B. Clerckx, R. Zhang, R. Schober, D. W. K. Ng, D. I. Kim, and H. V. Poor, "Fundamentals of wireless information and power transfer: From RF energy harvester models to signal and system designs," IEEE J. Sel. Areas Commun., vol. 37, no. 1, pp. 4-33, Jan. 2019.

[7] H. Ju and R. Zhang, "Throughput maximization in wireless powered communication networks," IEEE Trans. Wireless Commun., vol. 13, no. 1, pp. 418-428, Jan. 2014.

[8] Z. Gao, L. Dai, D. Mi, Z. Wang, M. A. Imran, and M. Z. Shakir, "MmWave massive-MIMO-based wireless backhaul for the 5G ultradense network," IEEE Wireless Commun., vol. 22, no. 5, pp. 13-21, Oct. 2015.

[9] X. Chen, C. Zhong, C. Yuen, and H. Chen, "Multi-antenna relay aided wireless physical layer security," IEEE Commun. Mag., vol. 53, no. 12, pp. 40-46, Dec. 2015.

[10] Renzo, Marco Di, et. al., "Smart radio environments empowered by reconfigurable AI meta-surfaces: an idea whose time has come," EURASIP J. Wirel. Commun. Netw., no. 129, pp. 1-20, May 2019.

[11] M. D. Renzo, A. Zappone, M. Debbah, M. Alouini, C. Yuen, J. D. Rosny, and S. Tretyakov, "Smart radio environments empowered by reconfigurable intelligent surfaces: How it works, state of research, and road ahead," to appear in IEEE J. Sel. Areas Commun., 2020. 
[12] C. Huang, S. Hu, G. C. Alexandropoulos, A. Zappone, C. Yuen, R. Zhang, M. Di Renzo, and M. Debbah, "Holographic MIMO surfaces for 6G wireless networks: Opportunities, challenges, and trends," to appear in IEEE Wireless Commun., 2020.

[13] Q. Wu and R. Zhang, "Intelligent reflecting surface enhanced wireless network via joint active and passive beamforming," IEEE Trans Wireless Commun., vol. 18, no. 11, pp. 5394-5409, Nov. 2019.

[14] Q. Wu, S. Zhang, B. Zheng, C. You, and R. Zhang, "Intelligent reflecting surface aided wireless communications: A tutorial," to appear in IEEE Trans. Commun., 2021.

[15] J. Yao and N. Ansari, "Joint content placement and storage allocation in C-RANs for IoT sensing service," IEEE Internet Things J., vol. 6, no. 1, pp. 1060-1067, Feb. 2019.

[16] W. Hao, M. Zeng, G. Sun, and P. Xiao, "Edge cache-assisted secure lowlatency millimeter-wave transmission," IEEE Internet Things J., vol. 7, no. 3, pp. 1815-1825, Mar. 2020.

[17] C. Huang, A. Zappone, G. C. Alexandropoulos, M. Debbah, and C. Yuen, "Reconfigurable intelligent surfaces for energy efficiency in wireless communication," IEEE Trans. Wireless Commun., vol. 18, no. 8 , pp. 4157-4170, Aug. 2019.

[18] Z. Chu, W. Hao, P. Xiao, and J. Shi, "Intelligent reflecting surface aided multi-antenna secure transmission," IEEE Wireless Commun. Lett., vol. 9, pp. 108-112, Jan 2020.

[19] H. Shen, W. Xu, S. Gong, Z. He, and C. Zhao, "Secrecy rate maximization for intelligent reflecting surface assisted multi-antenna communications," IEEE Commun. Lett., vol. 23, no. 9, pp. 1488-1492, Sep. 2019.

[20] X. Guan, Q. Wu, and R. Zhang, "Intelligent reflecting surface assisted secrecy communication: Is artificial noise helpful or not?" IEEE Wireless Commun. Lett., vol. 9, no. 6, pp. 778-782, Jun. 2020.

[21] C. Pan, H. Ren, K. Wang, W. Xu, M. Elkashlan, A. Nallanathan, and L. Hanzo, "Multicell MIMO communications relying on intelligent reflecting surfaces," IEEE Trans. Wireless Commun., vol. 19, no. 8, pp. 5218-5233, Aug. 2020.

[22] Q. Wu and R. Zhang, "Weighted sum power maximization for intelligent reflecting surface aided SWIPT," IEEE Wireless Commun. Lett., vol. 9, no. 5, pp. 586-590, May 2020.

[23] Q. Wu and R. Zhang, "Joint active and passive beamforming optimization for intelligent reflecting surface assisted SWIPT under QoS constraints," IEEE J. Sel. Areas Commun., vol. 38, no. 8, pp. 17351748, Aug. 2020.

[24] C. Pan, H. Ren, K. Wang, M. Elkashlan, A. Nallanathan, J. Wang, and L. Hanzo, "Intelligent reflecting surface aided MIMO broadcasting for simultaneous wireless information and power transfer," IEEE J. Sel. Areas Commun., vol. 38, no. 8, pp. 1719-1734, 2020.

[25] W. Hao, G. Sun, M. Zeng, Z. Zhu, Z. Chu, O. A. Dobre, and P. Xiao, "Robust design for intelligent reflecting surface assisted MIMOOFDMA terahertz communications," to appear in Internet Things $J$., 2021.

[26] S. Zhang and R. Zhang, "Intelligent reflecting surface aided multiple access: Capacity region and deployment strategy," in Proc. IEEE SPAWC, Atlanta, GA, USA, pp. 1-5, 2020.

[27] Y. Zheng, S. Bi, Y. J. Zhang, Z. Quan, and H. Wang, "Intelligent reflecting surface enhanced user cooperation in wireless powered communication networks," IEEE Wireless Commun. Lett., 2020.

[28] Z. Chu, P. Xiao, M. Shojafar, D. Mi, J. Mao, and W. Hao, "Intelligent reflecting surface assisted mobile edge computing for internet of things," IEEE Wireless Commun. Lett., pp. 1-5, 2020.

[29] G. S. Paschos, G. Iosifidis, M. Tao, D. Towsley, and G. Caire, "The role of caching in future communication systems and networks," IEEE J. Sel. Areas Commun., vol. 36, no. 6, pp. 1111-1125, Jun. 2018

[30] M. Tao, E. Chen, H. Zhou, and W. Yu, "Content-centric sparse multicast beamforming for cache-enabled cloud RAN," IEEE Trans. Wireless Commun., vol. 15, no. 9, pp. 6118-6131, Sept. 2016.

[31] Y. Fu, W. Wen, Z. Zhao, T. Q. S. Quek, S. Jin, and F. Zheng, "Dynamic power control for NOMA transmissions in wireless caching networks," IEEE Wireless Commun. Lett., vol. 8, no. 5, pp. 1485-1488, Oct. 2019.

[32] S. He, J. Ren, J. Wang, Y. Huang, Y. Zhang, W. Zhuang, and S. Shen, "Cloud-edge coordinated processing: Low-latency multicasting transmission," IEEE J. Sel. Areas Commun., vol. 37, no. 5, pp. 1144-1158, May 2019.

[33] D. Liu and C. Yang, "Energy efficiency of downlink networks with caching at base stations," IEEE J. Sel. Areas Commun., vol. 34, no. 4, pp. 907-922, Apr. 2016.

[34] J. Hu, W. Zhang, F. Wu, and K. Yang, "Simultaneous wireless content and power transfer in F-RAN: Caching, eRRH classification and beam- forming," IEEE Trans. Veh. Technol., vol. 69, no. 11, pp. 12992-13004, 2020.

[35] Z. Su, M. Dai, Q. Xu, R. Li, and S. Fu, "Q-Learning-based spectrum access for content delivery in mobile networks," IEEE Trans. Cogn. Commun. Netw., vol. 6, no. 1, pp. 35-47, Mar. 2020.

[36] Q. Wu and R. Zhang, "Beamforming optimization for wireless network aided by intelligent reflecting surface with discrete phase shifts," IEEE Trans. Commun., vol. 68, no. 3, pp. 1838-1851, 2020.

[37] Z. Chu, Z. Zhu, F. Zhou, M. Zhang, and N. Al-Dhahir, "Intelligent reflecting surface assisted wireless powered sensor networks for internet of things," IEEE Trans. Commun., vol. 69, no. 7, pp. 4877-4889, Jul. 2021.

[38] C. R. Valenta and G. D. Durgin, "Harvesting wireless power: Survey of energy-harvester conversion efficiency in far-field, wireless power transfer systems," IEEE Microw. Mag, vol. 15, no. 4, pp. 108-120, Jun. 2014.

[39] E. Boshkovska, D. W. K. Ng, N. Zlatanov, and R. Schober, "Practical non-linear energy harvesting model and resource allocation for SWIPT systems," IEEE Commun. Lett., vol. 19, no. 12, pp. 2082-2085, Dec. 2015.

[40] S. Wang, M. Xia, K. Huang, and Y.-C. Wu, "Wirelessly powered two-way communication with nonlinear energy harvesting model: Rate regions under fixed and mobile relay," IEEE Trans. Wireless Commun., vol. 16, no. 12, pp. 8190-8204, Dec. 2017.

[41] B. Lyu, P. Ramezani, D. T. Hoang, S. Gong, Z. Yang, and A. Jamalipour, "Optimized energy and information relaying in self-sustainable IRSempowered WPCN," IEEE Trans. Commun., vol. 69, no. 1, pp. 619-633, Jan. 2021.

[42] A. H. Mohsenian-Rad, V. W. S. Wong, J. Jatskevich, R. Schober, and A. Leon-Garcia, "Autonomous demand-side management based on game-theoretic energy consumption scheduling for the future smart grid," IEEE Trans. Smart Grid, vol. 1, no. 3, pp. 320-331, Dec. 2010.

[43] Z. Chu, P. Xiao, D. Mi, W. Hao, M. Khalily, and L.-L. Yang, "A novel transmission policy for intelligent reflecting surface assisted wireless powered sensor networks," IEEE J. Sel. Topics Signal Process., pp. 1$1,2021$.

[44] Y. Sun, P. Babu, and D. P. Palomar, "Majorization-minimization algorithms in signal processing, communications, and machine learning," IEEE Trans. Signal Process., vol. 65, no. 3, pp. 794-816, Feb. 2017.

[45] J. Song, P. Babu, and D. P. Palomar, "Sequence design to minimize the weighted integrated and peak sidelobe levels," IEEE Trans. Signal Process., vol. 64, no. 8, pp. 2051-2064, Apr. 2016.

[46] K. Alhujaili, V. Monga, and M. Rangaswamy, "Transmit MIMO radar beampattern design via optimization on the complex circle manifold," IEEE Trans. Signal Process, vol. 67, no. 13, pp. 3561-3575, Jul. 2019.

[47] X. Yu, J. Shen, J. Zhang, and K. B. Letaief, "Alternating minimization algorithms for hybrid precoding in millimeter wave mimo systems," IEEE J. Sel. Topics Signal Process., vol. 10, no. 3, pp. 485-500, Apr. 2016

[48] O. Ozdogan, E. Bjornson, and E. G. Larsson, "Intelligent reflecting surfaces: Physics, propagation, and pathloss modeling," IEEE Wireless Commun. Lett., vol. 9, no. 5, pp. 581-585, May 2020.

[49] A. Frville, "The multidimensional 0-1 knapsack problem: An overview," Eur. J. Oper. Res, vol. 155, no. 1, pp. 1 - 21, 2004.

[50] Q. Wu and R. Zhang, "Beamforming optimization for wireless network aided by intelligent reflecting surface with discrete phase shifts," IEEE Trans. Commun., vol. 68, no. 3, pp. 1838-1851, Mar. 2020. 